\title{
Computational Design and Analysis of a Transonic Natural Laminar Flow Wing for a Wind Tunnel Model
}

\author{
Michelle N. Lynde ${ }^{1}$ and Richard L. Campbell ${ }^{2}$ \\ NASA Langley Research Center, Hampton, Virginia, 23681
}

\begin{abstract}
A natural laminar flow (NLF) wind tunnel model has been designed and analyzed for a wind tunnel test in the National Transonic Facility (NTF) at the NASA Langley Research Center. The NLF design method is built into the CDISC design module and uses a NavierStokes flow solver, a boundary layer profile solver, and stability analysis and transition prediction software. The NLF design method alters the pressure distribution to support laminar flow on the upper surface of wings with high sweep and flight Reynolds numbers. The method addresses transition due to attachment line contamination/transition, Görtler vortices, and crossflow and Tollmien-Schlichting modal instabilities. The design method is applied to the wing of the Common Research Model (CRM) at transonic flight conditions. Computational analysis predicts significant extents of laminar flow on the wing upper surface, which results in drag savings. A $5.2 \%$ scale semispan model of the CRM NLF wing will be built and tested in the NTF. This test will aim to validate the NLF design method, as well as characterize the laminar flow testing capabilities in the wind tunnel facility.
\end{abstract}

\section{Nomenclature}

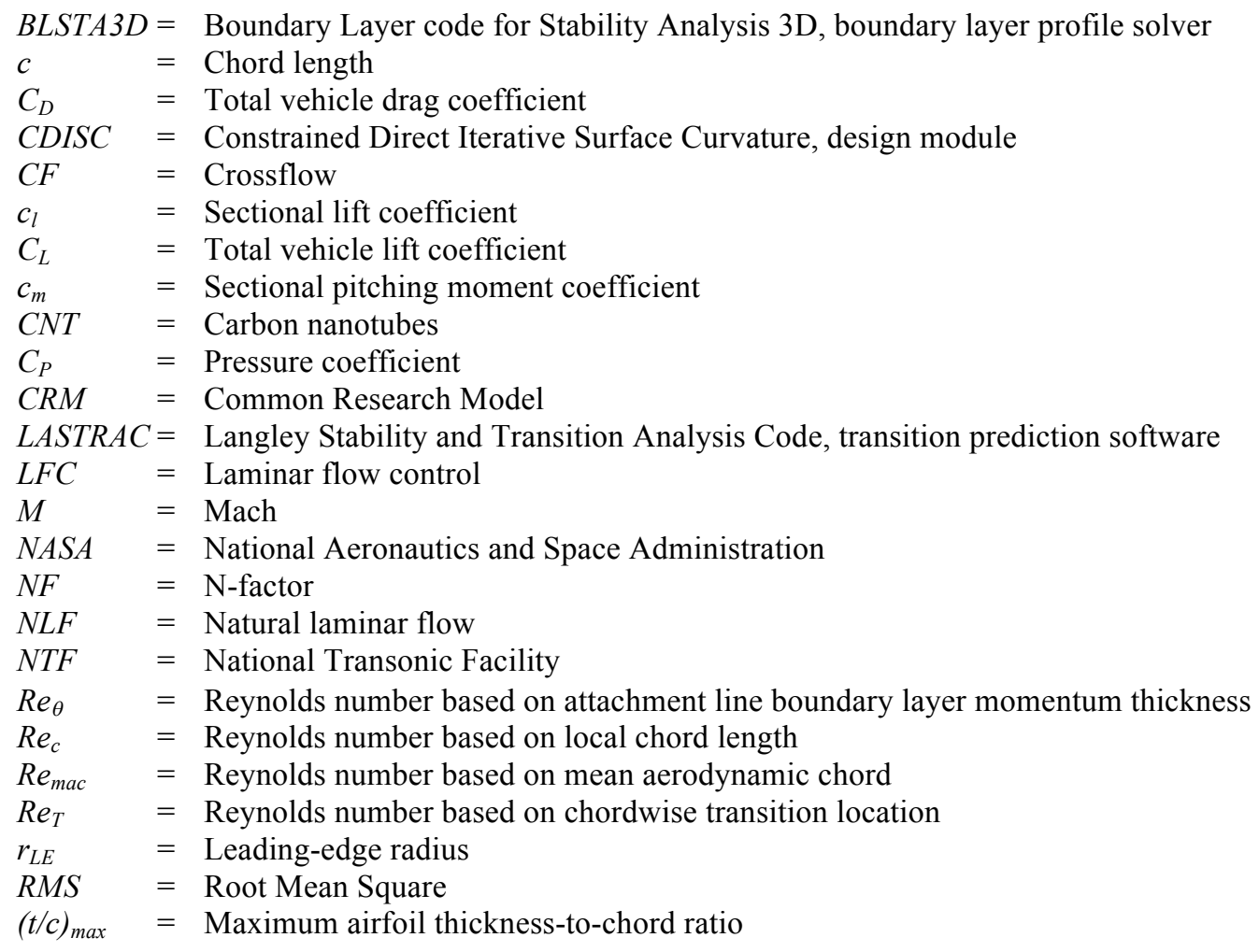

\footnotetext{
${ }^{1}$ Research Aerospace Engineer, Configuration Aerodynamics Branch, NASA Langley Resarch Center M/S 499 Hampton, VA 23681, AIAA Member.

${ }^{2}$ Senior Research Engineer, Configuration Aerodynamics Branch, NASA Langley Research Center M/S 499 Hampton, VA 23681, AIAA Associate Fellow.
} 
TetrUSS $=$ Tetrahedral Unstructured Software System, flow solver package

TS $=$ Tollmien-Schlichting

$T S P=$ Temperature sensitive paint

USM3D = Unstructured Mesh 3D, Navier-Stokes flow solver

$x / c=\mathrm{x}$-location nondimensionalized by local chord

$x 1, x 2, x 3=$ CDISC NLF constraint parameters corresponding to $\mathrm{x} / \mathrm{c}$ locations on pressure distributions

$z / c=$ z-location nondimensionalized by local chord

$\eta \quad=\quad$ Semispan location nondimensionalized by semispan length

$\Lambda_{L E} \quad=$ Leading-edge sweep

$\Lambda_{T E} \quad=$ Trailing-edge sweep

\section{Introduction}

S environmental concerns grow and gas prices rise, the need for more efficient transonic transports becomes increasingly prevalent. Industry and research partners work to keep up with growing air traffic demand, while searching for promising new drag-saving technology to incorporate in future vehicles. One major contributor to a vehicle's overall drag is skin friction drag, and one promising technique for reducing skin friction drag is by sustaining natural laminar flow (NLF). Presently, applications of NLF are limited to vehicle components with small leading-edge sweep and low Reynolds number because increasing either parameter commonly increases the growth of modal instabilities that lead to transition. Figure 1 shows the present NLF region based on transition Reynolds number $\left(\operatorname{Re}_{\mathrm{T}}\right)$ and leading-edge sweep $\left(\Lambda_{\mathrm{LE}}\right)$. This figure shows the F-14 Variable Sweep Transition Flight Experiment results, which is the highest published combination of transition Reynolds number and leading-edge sweep using NLF technology ${ }^{1}$. This represents the current boundary of NLF. Aircraft components with Reynolds number or sweep beyond this region would require using a laminar flow control (LFC) system to sustain laminar flow. LFC systems often introduce performance or operational penalties related to added weight or system complexity, which have limited its application in aircraft. NLF technology is proving to be an attractive drag-saving option for future aircraft, and is currently being flown on some vehicle components with low sweep and/or Reynolds number. The HondaJet wing, Boeing 787 nacelle, and Boeing 737 Max winglet support various extents of $\mathrm{NLF}^{2,3}$. Supporting NLF on the main wing of a typical transonic

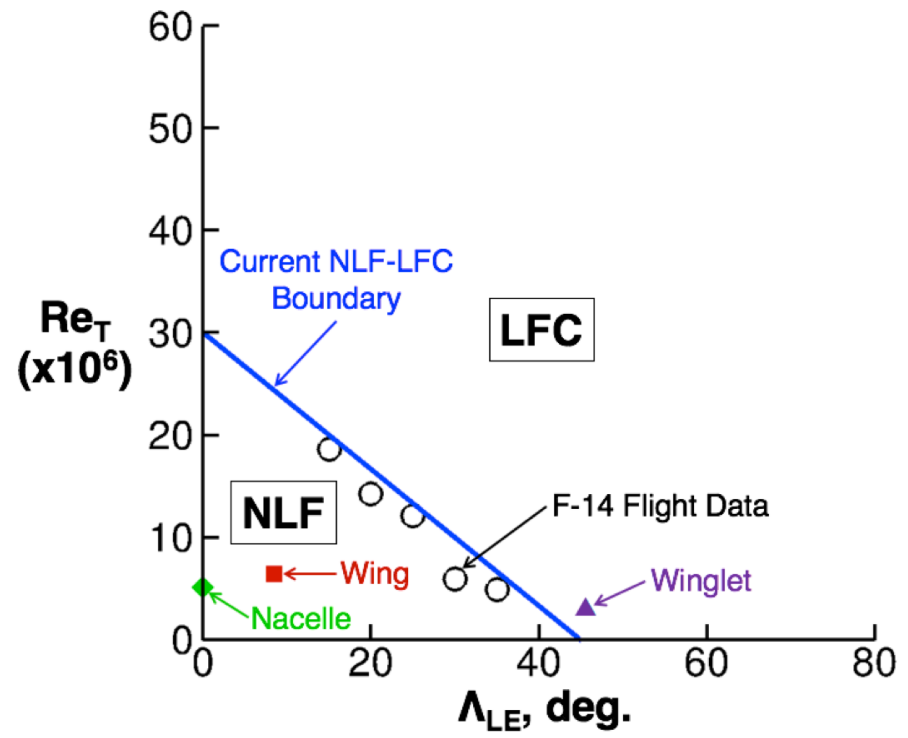

Figure 1. Current NLF-LFC boundary in terms of transition Reynolds number $\left(\operatorname{Re}_{\mathrm{T}}\right)$ and leading-edge sweep $\left(\Lambda_{\mathrm{LE}}\right)$.

transport vehicle offers the potential to significantly reduce the overall drag, translating to aerodynamic performance benefits and reduced operating cost of the aircraft. A new computationally based NLF design method is being developed that predicts significant regions of laminar flow on configurations with high leading-edge sweep and high Reynolds numbers.

Like most new flight technology, the process of incorporating NLF into vehicles involves validating designs in wind tunnels. Laminar flow testing presents a unique challenge due to its dependence on environments. Modern wind tunnels often have significantly higher turbulence levels than flight conditions, which directly impacts the extent of laminar flow. An NLF test has been proposed in the National Transonic Facility (NTF) at the National Aeronautics and Space Administration (NASA) Langley Research Center to better characterize the facility's laminar flow testing capability. This includes quantifying the tunnel critical N-factor (NF) for both crossflow (CF) and TollmienSchlichting (TS) instabilities, determining any upper limits of Reynolds number for laminar flow visualization based on flow quality, applying innovative laminar flow visualization techniques at cryogenic conditions, and defining best 
practices for surface finish and manufacturing requirements. In addition to the testing-related goals, the proposed NTF test will aim to confirm the predicted extents of laminar flow of a new computationally based NLF design method.

\section{Design of the Wind Tunnel Model}

The proposed wind tunnel test will focus on quantifying laminar flow capabilities and visualization techniques in the NTF, as well as validating the extents of laminar flow predicted by the NLF design method. These test objectives resulted in a primary design goal of achieving significant extents of laminar flow on a swept wing in a wind tunnel environment. Aerodynamic performance is monitored throughout the model design process, but is not the driving design objective. The present section will discuss the design and analysis approach, including computational tools employed and details of the design method, as well as report the newly designed wind tunnel model's laminar flow and performance characteristics.

\section{A. Design and Analysis Approach}

The NLF design method is used in this application to reshape a transonic transport wing with high sweep and high Reynolds number in order to obtain significant extents of laminar flow on the upper surface. A suite of computational tools is used in the design and analysis of the wing. These tools enable the manipulation of the wing geometry and the evaluation of transition characteristics.

1. Computational Tools

The computational tools used in this NLF design work include a flow solver, a design module, and boundary layer stability analysis and transition prediction software. The flow chart in Figure 2 illustrate the NLF design and analysis framework. In this application, the transition prediction loop is not performed with each design loop, but rather once a completed design is obtained. This approach saves computational time and resources.

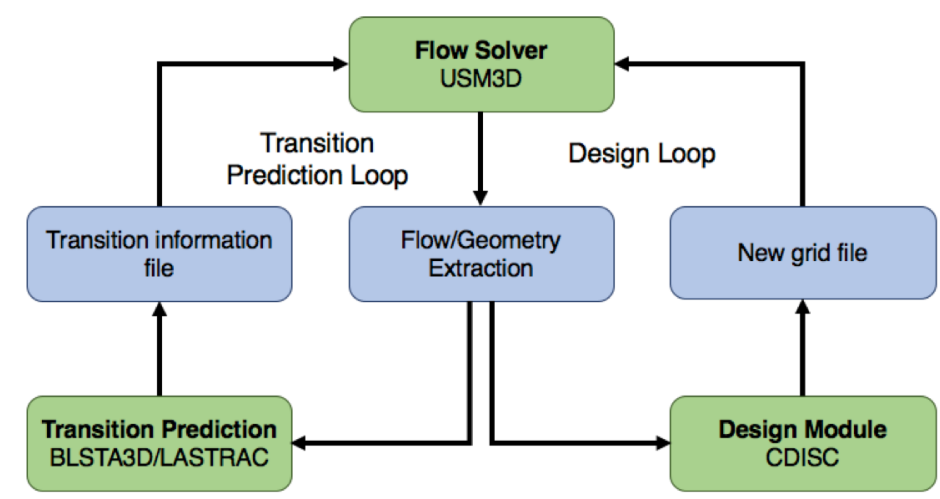

Figure 2. Flow chart of the NLF design and analysis framework.

The flow solver utilized is USM3D (Unstructured Mesh 3D), a cell-centered, finite-volume Navier-Stokes code that is part of TetrUSS (Tetrahedral Unstructured Software System $)^{4}$. To generate the tetrahedral cell grid used with USM3D, a triangular surface mesh is generated, after which viscous grid cells are built using an advancing layers method, followed by the remaining inviscid portion using an advancing front method. For this application, the grids have a $\mathrm{y}^{+}=0.5$ for the first cell off the surface, approximately 30 viscous layers, and further refined leading edges. The surface grid points were aligned along several chordwise stations across the span, which removed curvature noise caused by interpolating between grid points, and provided smoother curvature information to our design module. The flow solutions were obtained using the Spalart-Allmaras turbulence model in regions of turbulent flow. The forced laminarization feature within USM3D was used to model laminar flow features ahead of a designated transition front, such as reduced skin friction and boundary layer thickness, which is essential to evaluating an NLF design.

The flow solver is coupled with a design module, CDISC (Constrained Direct Iterative Surface Curvature) ${ }^{5}$. CDISC is a knowledge-based design tool, which is used in this application to alter the geometry to produce the desired NLF pressure architecture. CDISC flow constraints allow designing to common engineering variables, such as span load, section lift and/or pitching moment coefficients, and shock strength. Similarly, geometry constraints are available to address requirements from other disciplines such as structures and manufacturing, including: thickness, curvature, volume, and leading-edge radius. In this application of the design module, CDISC automatically produces target pressures that support significant extents of laminar flow based on the current analysis pressures. Previous examples of the NLF design capability within CDISC have been reported for both transonic and supersonic configurations ${ }^{6,7}$. Additional detail of the CDISC NLF design process is described below.

The boundary layer stability analysis and transition prediction software is comprised of two main codes: BLSTA3D (Boundary Layer code for Stability Analysis 3D) ${ }^{8}$ and LASTRAC (Langley Stability and Transition Analysis Code $)^{9}$. Boundary layer profile information is calculated from streamwise pressure distributions at several 
stations on the wing by the BLSTA3D code. This code is not a fully-3D boundary layer solver, so a conical flow approximation was used to account for sweep and taper in this transonic wing example. This code was also used to evaluate the attachment line state by calculating the Reynolds number based on attachment line boundary layer momentum thickness, $\mathrm{Re}_{\theta}$. The boundary layer profiles from BLSTA3D are analyzed using the LASTRAC software package, which is a set of physics-based codes that allows the user to perform stability analysis with a range of fidelity. For this application, the $\mathrm{e}^{\mathrm{N}}$ method was employed using linear stability theory with the fixed-beta method and includes compressibility effects. For transonic wing designs, both CF and TS modal instability growths are calculated and do not include curvature effects. For CF analysis, 20 wavenumbers (betas) are analyzed at $0 \mathrm{~Hz}$ frequency and are adjusted to capture the highest growing NF values. Similarly, for TS analysis, 20 frequencies are analyzed at 0 wavenumber and adjusted for maximum growth. The current method of transition prediction is evaluated and discussed in more detail in the companion paper, "Building a Practical Natural Laminar Flow Design Capability". A critical NF, which corresponds to the NF value where transition should occur, is prescribed and used to create the transition front. While the design method can design to different CF and TS critical NF, for this application the same value was used for both instabilities.

\section{CDISC Design Method}

The design approach reported here focuses on addressing three mechanisms of boundary layer transition. The first of these is Görtler vortices, which are generated in regions of negative surface curvature ${ }^{10}$. To guard against this type of transition, a curvature constraint within CDISC is implemented in the design process to ensure there is no negative surface curvature in the region where NLF is expected. The second transition mechanism is attachment line contamination and transition. Attachment line contamination occurs when a turbulent boundary layer from the fuselage runs onto the leading edge of a swept wing, causing the wing attachment line to be turbulent, and resulting in total loss of laminar flow over the wing. Attachment line transition occurs when the geometry features along the attachment line, such as sweep and radius, are susceptible to disturbance growth and cause the attachment line to be turbulent. Both attachment line contamination and transition are evaluated in the present work using Poll's criteria based on attachment line $\mathrm{Re}_{\theta}{ }^{11}$, which is calculated from the boundary layer profile solver, BLSTA3D. Further detail on the design method employed for this model to address attachment line contamination and transition is discussed in the following subsection. The final transition mechanism is modal instabilities. For transonic flow, this transition mechanism can be further broken down into two categories: Crossflow (CF) and Tollmien-Schlichting (TS) instabilities. In order to sustain NLF, the present design method alters wing pressures to control CF and TS growth below a prescribed critical NF. All three of the aforementioned transition mechanisms are considered in the design of the wind tunnel model.

The CDISC NLF design method takes an existing turbulent pressure distribution and alters it to suppress $\mathrm{CF}$ and TS NF growth in order to sustain NLF. Within the CDISC NLF constraint, there are three parameters that can be adjusted for each configuration. Figure 3 illustrates these three parameters on a sample pressure distribution, and are labeled $\mathrm{x} 1, \mathrm{x} 2$, and $\mathrm{x} 3$. The first parameter, $\mathrm{x} 1$, is adjusted to control CF growth, which is primarily dominant in the leading-edge region. The rapid acceleration (ending at $\mathrm{x} 1$ ) is followed by a short region of zero pressure gradient (ending at $\mathrm{x} 2$ ), and is the key feature in damping CF growth on wings with higher sweep and Reynolds numbers. The $\mathrm{x} 1$ location is correlated to the maximum $\mathrm{CF} \mathrm{NF}$ growth; the more forward $\mathrm{x} 1$ is located, the more aggressive the $\mathrm{CF}$ damping. The $\mathrm{x} 1$ location is selected internally within CDISC such that the maximum CF NF is 2-3 units below the specified

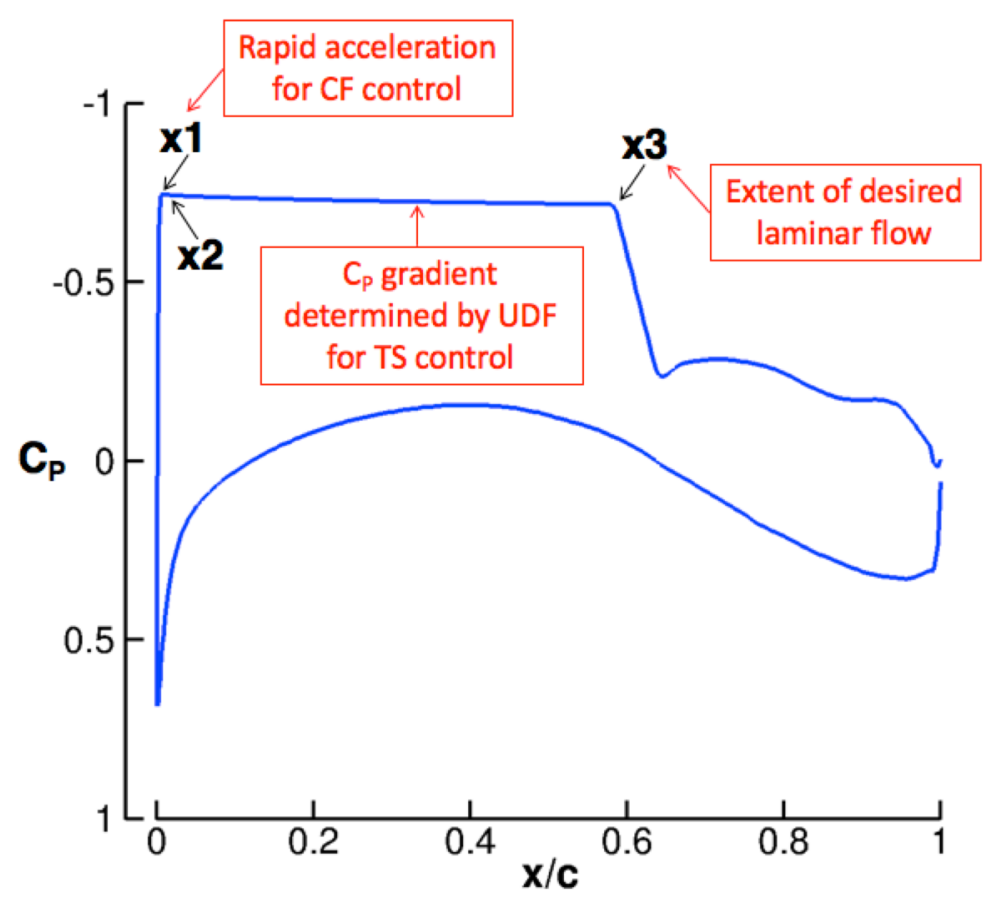

Figure 3. Sample target pressure distribution generated by CDISC for NLF showing three design parameters: $x 1, x 2$, and $x 3$. 
critical NF level. The final two parameters, labeled $\mathrm{x} 2$ and $\mathrm{x} 3$, are used to control TS growth. The midchord slope between the $\mathrm{x} 2$ and $\mathrm{x} 3$ locations is determined within the CDISC NLF constraint by an equation called the Universal Damping Function (UDF). The UDF is adjusted to damp TS growth, keeping the NF growth below a designated critical NF until the desired chordwise location. The $\mathrm{x} 3$ parameter represents the extent of the desired laminar flow; pressures aft of $\mathrm{x} 3$ are blended back to the current analysis pressures. These aft pressures are adjusted where necessary to maintain a reasonable closure and, for transonic flow, to match a prescribed pitching-moment value for good performance based on empirical studies. The pressure level on the upper surface is adjusted to meet a specified sectional lift coefficient to match the desired spanloading. The lower surface target pressures are generally unaltered from the current analysis pressures, though some adjustments are made in the aft cove region to obtain the desired pitching moment and ensure reasonable aft thickness. Examples and additional details of the CDISC NLF constraint have been published in previous reports ${ }^{6,7}$.

\section{B. Model Design Results}

The wind tunnel model is a semispan model that will utilize an existing semispan fuselage from a previous NTF test ${ }^{12}$. The fuselage will be retrofitted with the new NLF wing and a wing-fuselage fairing. The design characteristics of the wing are presented in this subsection.

\section{Design Condition}

The configuration selected as the baseline for the wind tunnel model is the Common Research Model (CRM), shown in Figure 4. The CRM was designed originally to be an open geometry representation of a generic transport vehicle and has been the subject of several studies ${ }^{13}$. The cruise design conditions for the CRM are a Mach number of 0.85 , a lift coefficient of 0.5 , and a Reynolds number of 40 million based on the mean

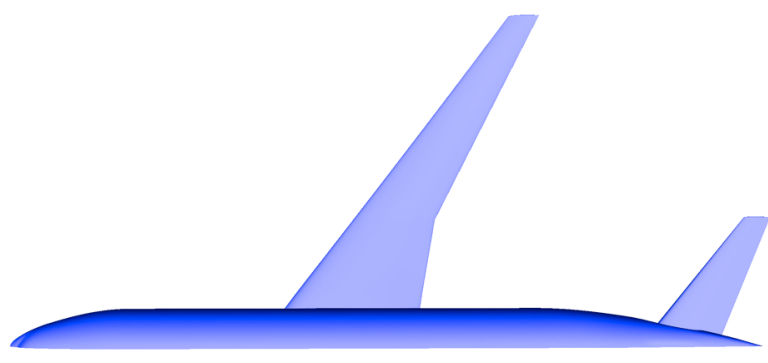

Figure 4. Planform view of the Common Research Model, which is used as the baseline configuration for the NLF wind tunnel model. aerodynamic chord. For this wind tunnel model, the design condition Reynolds number based on mean aerodynamic chord was reduced to 30 million because this was the highest Reynolds number tested previously ${ }^{14}$ and likely to be the upper limit of good visualization of laminar flow extent based on previous NLF testing in the facility $^{15}$. The wind tunnel model will be a $5.2 \%$ scale model of the CRM with the new NLF wing. Only the upper surface of the wing is designed to support NLF. The wing upper surface offers the largest potential for skin friction drag savings and it is assumed that the lower surface on a flight vehicle would transition due to steps or gaps from equipment, such as maintenance access panels or Krueger flaps. The CRM was also used as the baseline configuration for the transonic example of the CDISC NLF design method in a previous paper ${ }^{6}$. Several differences exist in the design approach between the previous example and the current wind tunnel model relating to addressing attachment line contamination, the critical NF, and manufacturing considerations.

The method for addressing attachment line contamination for the wind tunnel model is unique to this design. The previous transonic CRM example primarily utilized small leading-edge radii to reduce the Reynolds number based on attachment line momentum thickness, $\mathrm{Re}_{\theta}$, below Poll's limit of 100 for avoiding attachment line contamination. This technique proved challenging inboard and the final design did not have $\operatorname{Re}_{\theta} \leq 100$ until halfway out the span. Additionally, the design using the small leading-edge radii technique is more sensitive to off-design conditions, as changing the angle of attack causes the attachment point to shift and often moves off the small radius location. For the current wind tunnel model design, the method selected to address attachment line contamination is unsweeping the inboard section of the wing leading edge. This technique is thought to be more robust off design and provides additional suppression of $\mathrm{CF}$ instabilities inboard, both of which are advantageous in a wind tunnel environment. A new CDISC flow constraint was created in order to reduce the inboard sweep to locally meet the attachment line criteria on the $\mathrm{Re}_{\theta}$. Figure 5 shows the new wing-fuselage juncture, where the inboard $10 \%$ of the span has a reduced leading-edge sweep creating a "reverse fillet". For the baseline CRM, the wing has a constant leading-edge sweep of $37.3^{\circ}$. The new wind tunnel model will have a minimum sweep at the juncture of $12.9^{\circ}$, gradually increases the sweep as determined by the local $\operatorname{Re}_{\theta}$ value, and resumes the original $37.3^{\circ}$ at $10 \%$ span. Figure 6 shows the effects of this 
reduced sweep on attachment line $\mathrm{Re}_{\theta}$. The area of reduced sweep, between $\eta=0.10-0.20$, successfully reduces the attachment line $\mathrm{Re}_{\theta}$ below the contamination limit of 100 . Outboard of this section, the $\mathrm{Re}_{\theta}$ requirement is relaxed to meet the attachment line transition criteria of $\mathrm{Re}_{\theta} \leq 235$. The reduction of sweep at the juncture reduces the root chord by approximately $8 \%$, but the maximum thickness was maintained in order to accommodate future structural requirements or landing gear. Elsewhere on the wing, the maximum thickness-tochord ratio was held constant during the design. An evaluation of several design methods used to address attachment line contamination can be found in the companion paper, "Building a Practical Natural Laminar Flow Design Capability”.

For the wind tunnel model, the critical NF that the wing was designed to has been reduced from the previous transonic example. The motivation for reducing the design critical NF is that a wind tunnel

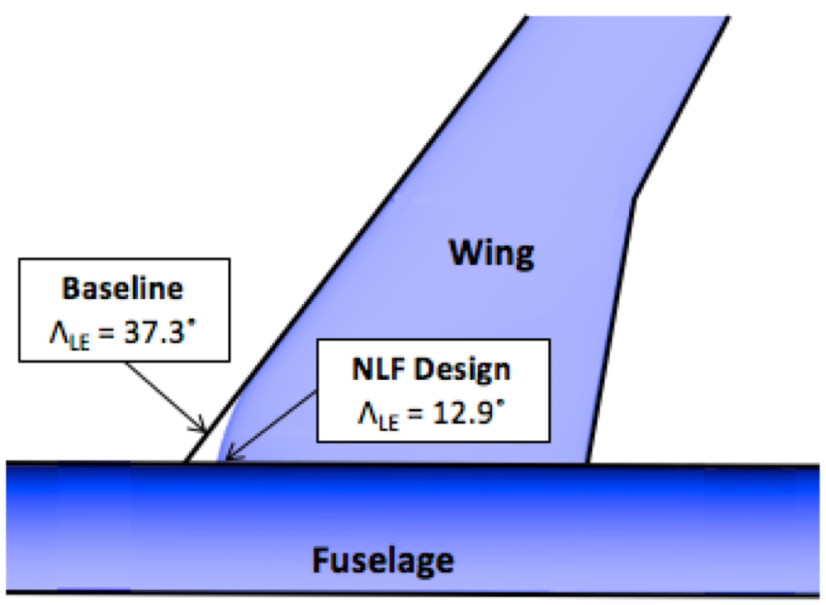

Figure 5. Planform view of the wing-fuselage juncture showing the reduced leading-edge sweep $\left(\Lambda_{L E}\right)$ of the NLF Design, which is used to control attachment line contamination. environment has a much lower critical NF than flight conditions. Literature reports a range of 9 to 14 for flight critical $\mathrm{NF}^{11,16}$, so the previous CRM example was designed for a critical NF of 13 to show the potential of the NLF method in a flight environment. However, the NTF is estimated to have a critical NF of approximately 6. As mentioned previously, the CDISC NLF design method adjusts the pressure distributions to keep CF and TS growth below a designated critical NF. Reducing the design critical NF requires the CF and TS growth to be damped more aggressively with the rapid acceleration pushed more forward and an increasingly favorable midchord pressure gradient, which often results in higher drag penalties. An attempt to reduce the design critical NF to the estimated NTF value of 6 was previously made and produced a geometry that had higher drag penalties and unrealistic geometries ${ }^{6}$. For the present work, a critical NF of 10 is used for the wind tunnel model design, which is more conservative within the reported

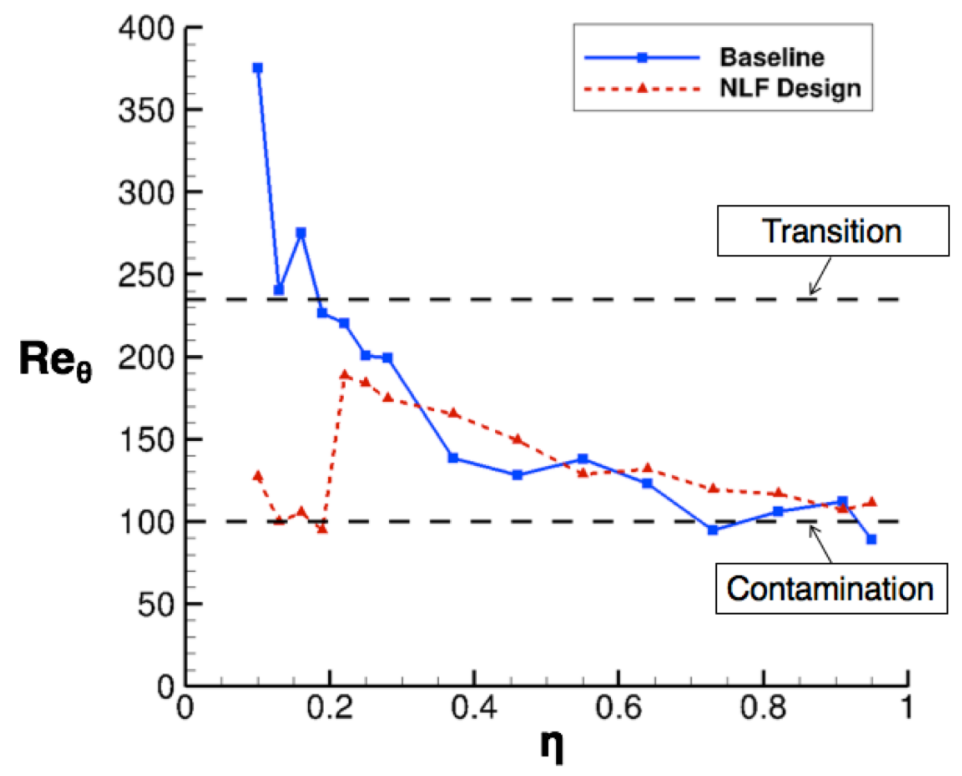

Figure 6. Attachment line $\operatorname{Re}_{\theta}$ versus semispan location $(\eta)$ of the Baseline and NLF Design configurations. The limits for transition $\left(\operatorname{Re}_{\theta} \leq 235\right)$ and contamination $\left(\operatorname{Re}_{\theta} \leq 100\right)$ are shown. The plot shows the suppressed $\operatorname{Re}_{\theta}$ values inboard on the NLF Design over the region of reduced leading-edge sweep. flight environment range of critical NF, but avoids incurring the additional penalties and challenges seen in the previous report when designing to the NTF critical NF. The additional CF and TS damping that will be needed in the NTF to keep instability growth below a critical NF of 6 will come from reducing the test Reynolds number. Additional details regarding testing strategy in the NTF are discussed in the following section.

Designing for a wind tunnel model necessitated additional attention to manufacturing considerations. The previous transonic CRM example was primarily used as a theoretical demonstration of the potential of the NLF design method. Additional attention was needed to maintain a practical final design for the wind tunnel model. This requirement resulted in implementing twist constraints and spanwise smoothing in the design process. The wind tunnel model also had curvature constraints to keep moderate curvature levels, which help mitigate off design penalties. 
During the design process, sustaining NLF was the primary goal. However, additional design constraints were added to maintain a realistic design and keep performance acceptable. As mentioned, maximum thickness was held constant across the span, and only the upper surface of the wing is designed for laminar flow. The spanwise loading and pitching moment distribution maintained similar characteristics throughout the design. Sixteen spanwise stations were used during the design process, shown in Figure 7. The flow solution of the configuration was obtained with appropriate extents of laminar flow modeled in the flow solver. The stations are located approximately every $10 \%$ span, with increased resolution (approximately 3\% span) surrounding the break in leading-edge sweep. Stability analysis was typically performance at each of the 16 design stations. Results will be shown from six spanwise stations in this report, highlighted and numbered in Figure 7. Table 1 provides additional details about each station. The wind tunnel model will be built to $5.2 \%$ scale.

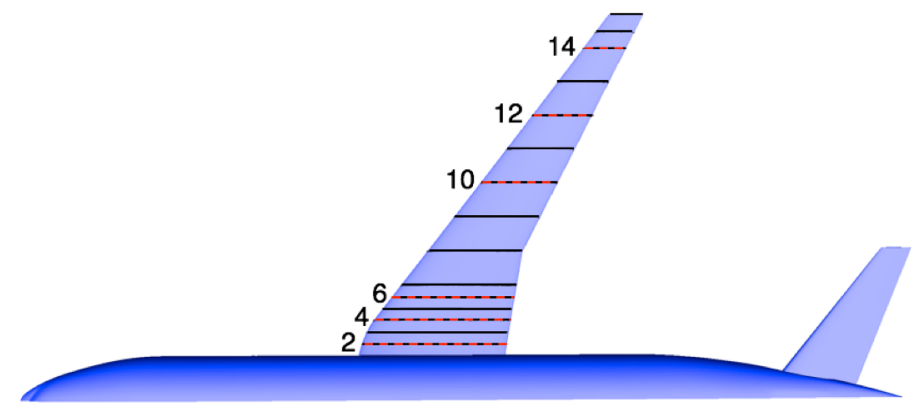

Figure 7. Planform view of the NLF Design configuration showing the 16 design stations. Results are shown from the 6 stations that are highlighted and numbered.

Table 1. Station details for the 6 example stations.

\begin{tabular}{|c|c|c|c|c|c|c|}
\hline Station & $\mathbf{2}$ & $\mathbf{4}$ & $\mathbf{6}$ & $\mathbf{1 0}$ & $\mathbf{1 2}$ & $\mathbf{1 4}$ \\
\hline $\boldsymbol{\eta}$ & 0.13 & 0.19 & 0.25 & 0.55 & 0.73 & 0.91 \\
\hline Chord (ft.) & 35.5 & 33.8 & 30.5 & 19.6 & 15.3 & 11.1 \\
\hline $\mathbf{R e}_{\mathbf{c}}$ (million) & 46.3 & 44.1 & 39.8 & 25.5 & 20.0 & 14.4 \\
\hline$\Lambda_{\mathbf{L E}}$ (deg.) & 16.5 & 33.0 & 37.3 & 37.3 & 37.3 & 37.3 \\
\hline$\Lambda_{\text {TE }}$ (deg.) & 10.0 & 10.0 & 10.0 & 27.3 & 27.3 & 27.3 \\
\hline
\end{tabular}

The primary approach to delaying transition involves altering the pressure distribution. Figure 8 compares the Baseline pressure distributions (blue, solid) to the final NLF Design pressure distribution (red, dashed) at the 6 example stations. The corresponding changes in airfoil geometry are also shown. Local twist changes have been removed in order to more clearly identify the changes in airfoil shape. Station 2 takes advantage of the CF suppression from the reduced leading-edge sweep, which makes the acceleration near the leading edge less aggressive. The pressure gradients required to keep TS growth below a critical NF of 10 resulted in the NLF Design shock strength increasing from the Baseline configuration. The lower surface and aft loading of each station was adjusted in order to keep the local lift coefficient and pitching moment coefficient similar to the Baseline configuration, but with slightly more aft loading inboard to reduce shock strengths. The NLF Design inboard airfoils required smaller leading-edge radii in order to obtain the rapid acceleration needed for $\mathrm{CF}$ suppression and to reduce the attachment line $\mathrm{Re}_{\theta}$, but the outboard airfoils required little change to obtain the NLF pressure distribution. The stability analysis results for both TS and CF analysis at the 6 example stations of the NLF Design configuration are shown in Figure 9. The black dashed line represents the critical NF; anytime the NF growth exceeds this limit, the flow is considered turbulent. These results were obtained using the analysis method outlined in the above "Design and Analysis Approach" subsection. These stability analysis plots show successful delay of transition to the designated chordwise location, typically transitioning just ahead of the shock around $\mathrm{x} / \mathrm{c}=0.60$. The transition front on the wing planform is shown in Figure 10. The NLF Design is predicted to support approximately $56 \%$ laminar flow based on surface area of the upper surface. 

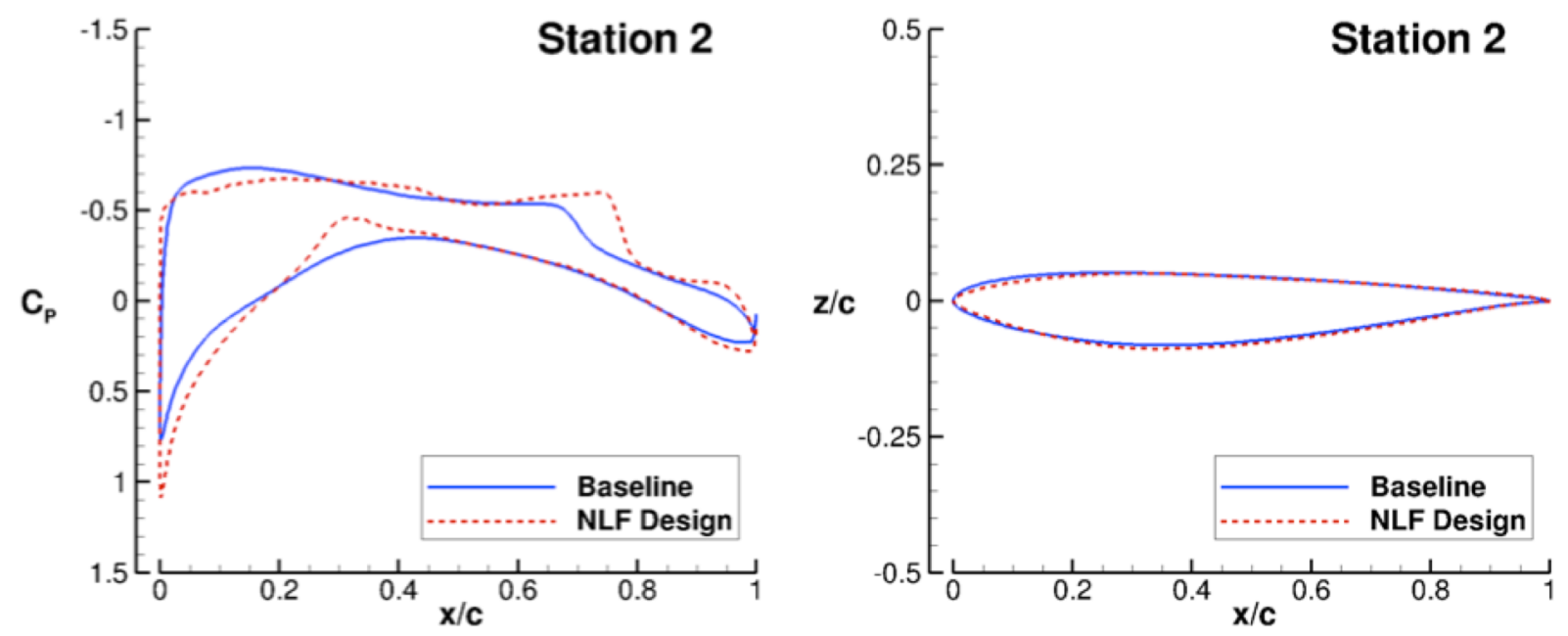

a) Station 2 pressure distribution (left) and airfoil geometry (right).
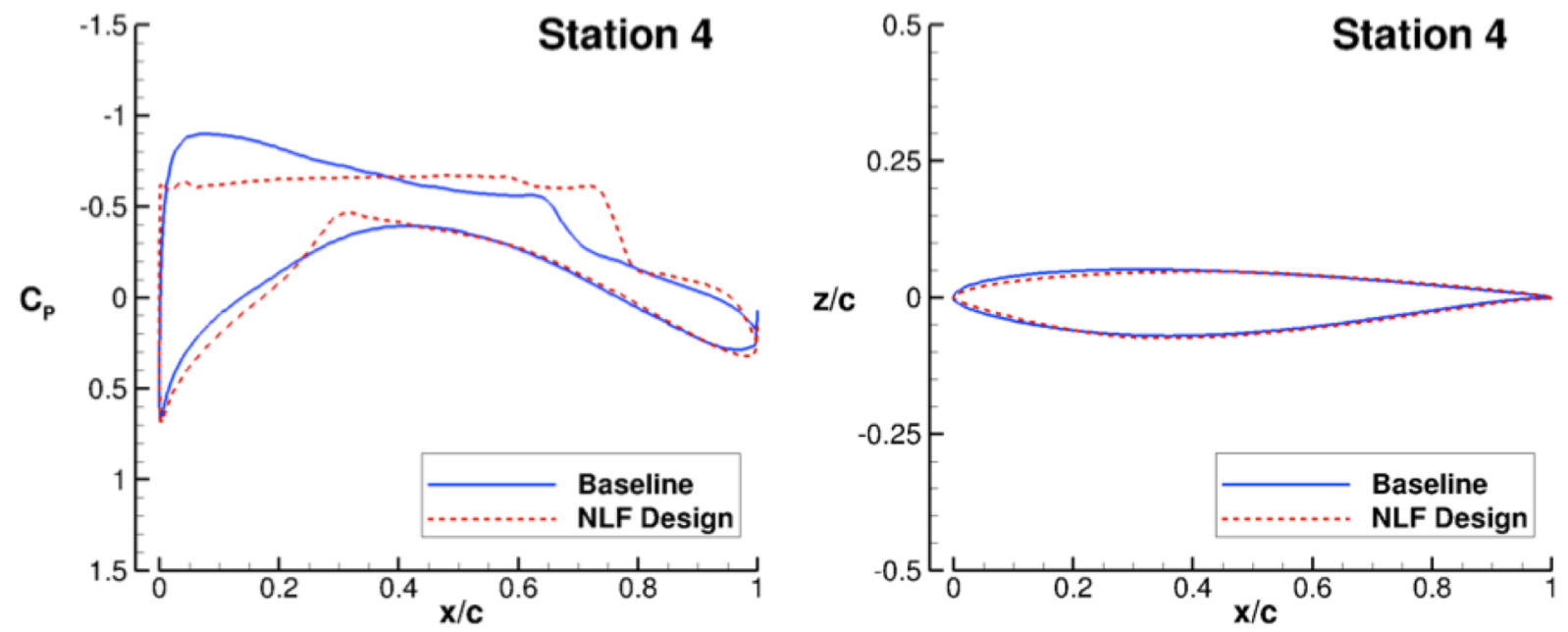

b) Station 4 pressure distribution (left) and airfoil geometry (right).
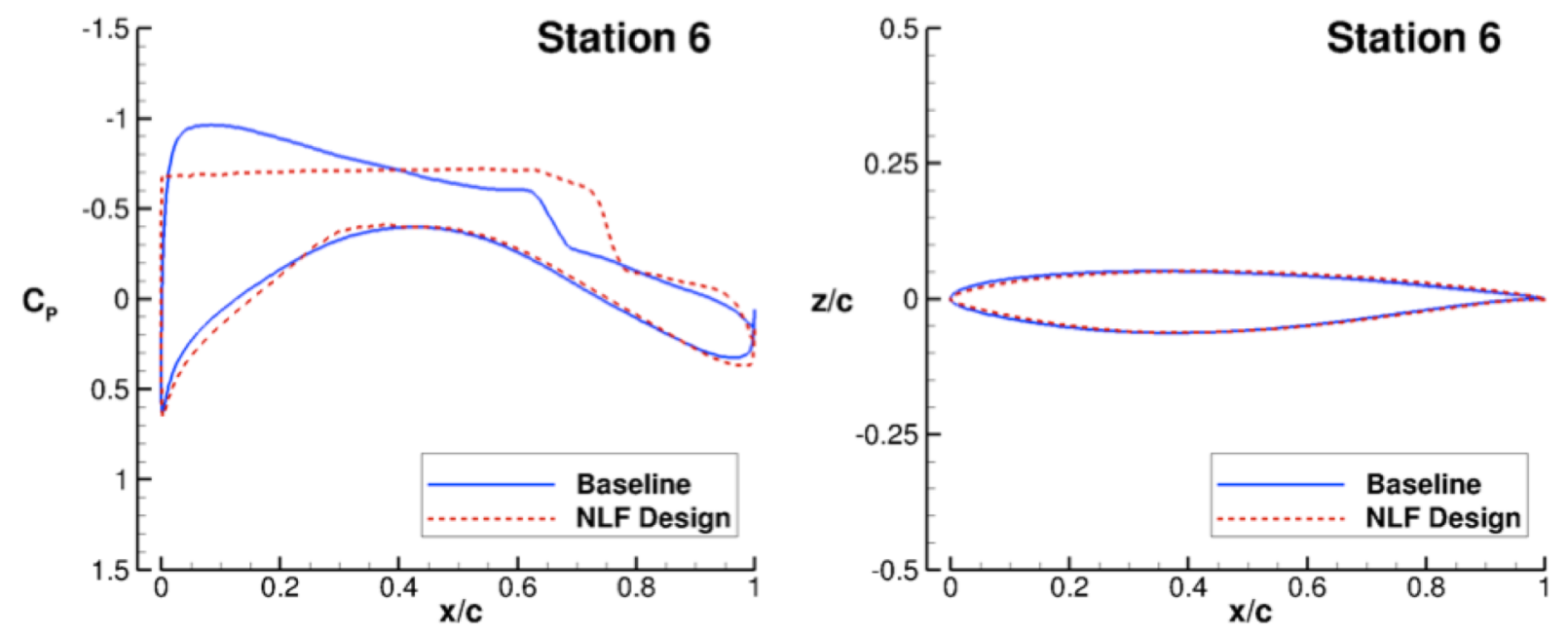

c) Station 6 pressure distribution (left) and airfoil geometry (right).

Figure 8. Comparison of the Baseline (blue, solid) and the NLF Design (red, dashed) configurations, showing pressure distributions (left) and airfoil geometry (right), analyzed at $M=0.85, C_{L}=0.5, \operatorname{Re}_{m a c}=30 \times 10^{6}$. 

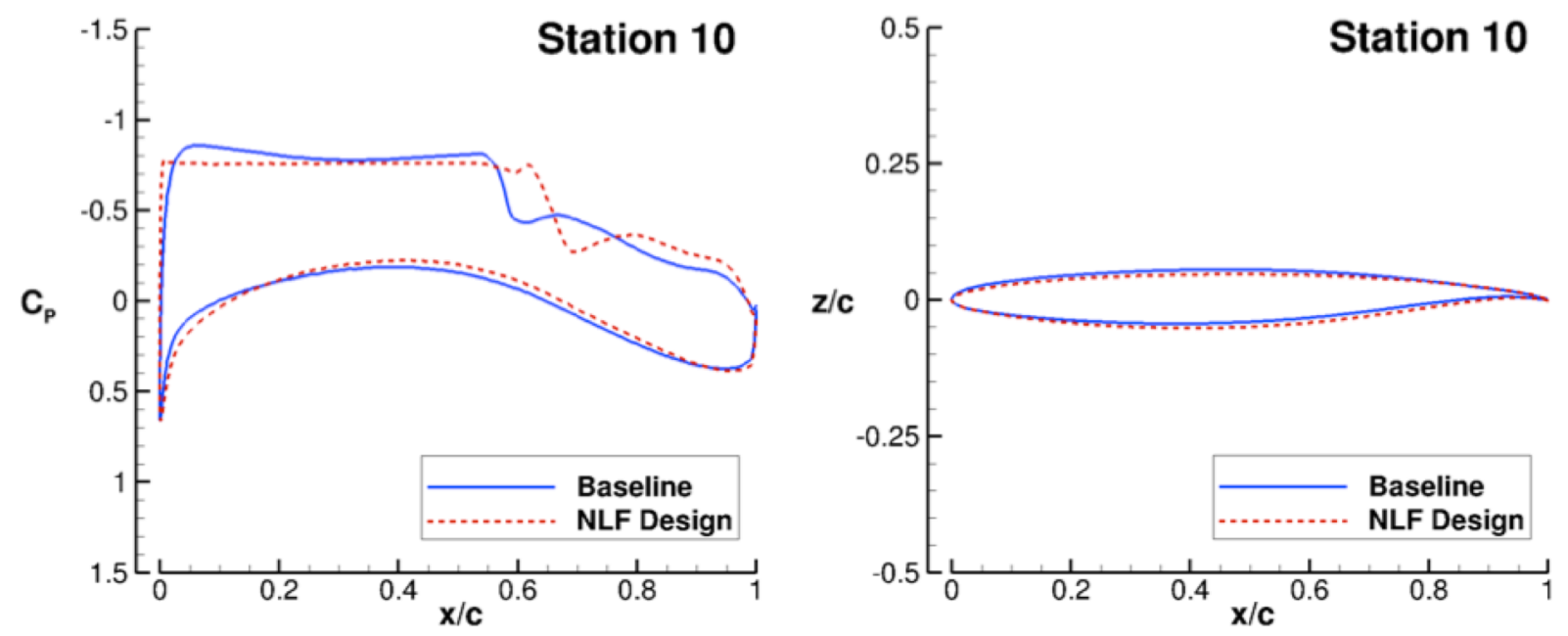

a) Station 10 pressure distribution (left) and airfoil geometry (right).
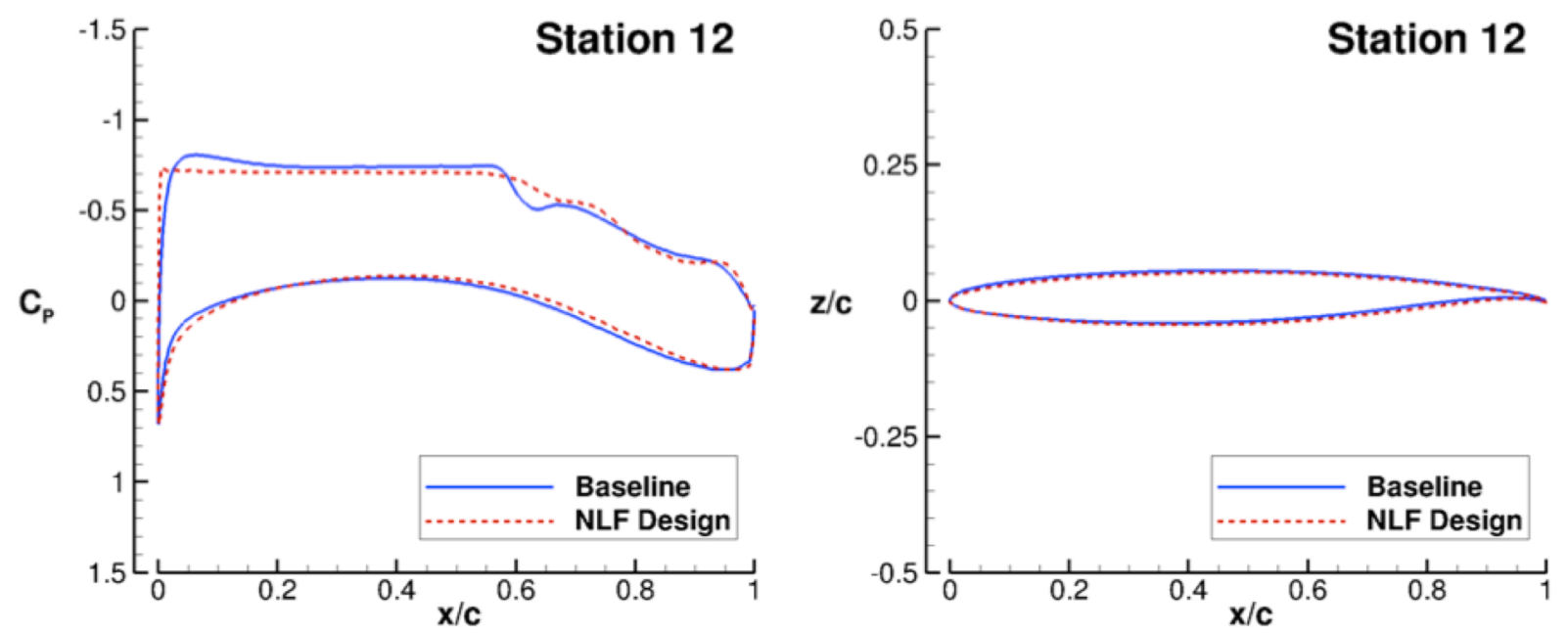

b) Station 12 pressure distribution (left) and airfoil geometry (right).
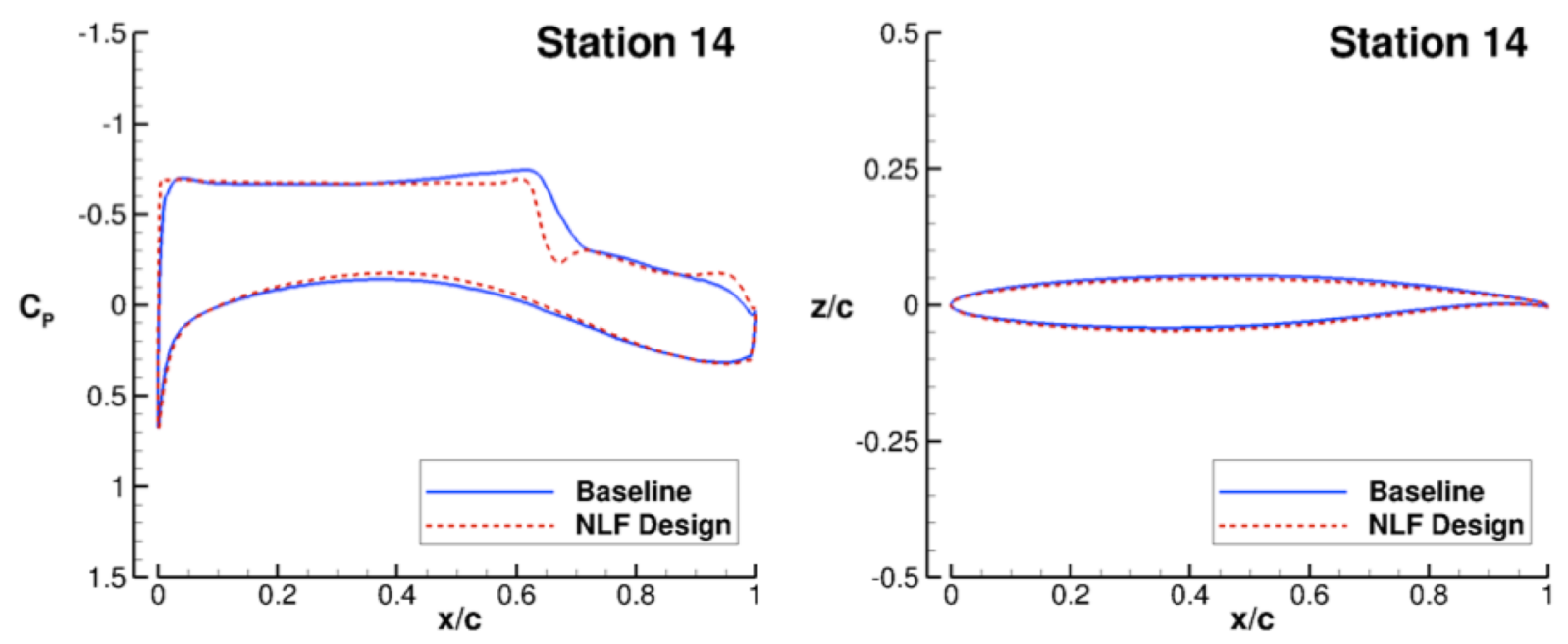

c) Station 14 pressure distribution (left) and airfoil geometry (right).

Figure 8 (continued). Comparison of the Baseline (blue, solid) and the NLF Design (red, dashed) configurations, showing pressure distributions (left) and airfoil geometry (right), analyzed at $M=0.85, C_{L}=0.5$, $\operatorname{Re}_{\text {mac }}=30 \times 10^{6}$.

9

American Institute of Aeronautics and Astronautics 


\section{Station 2}
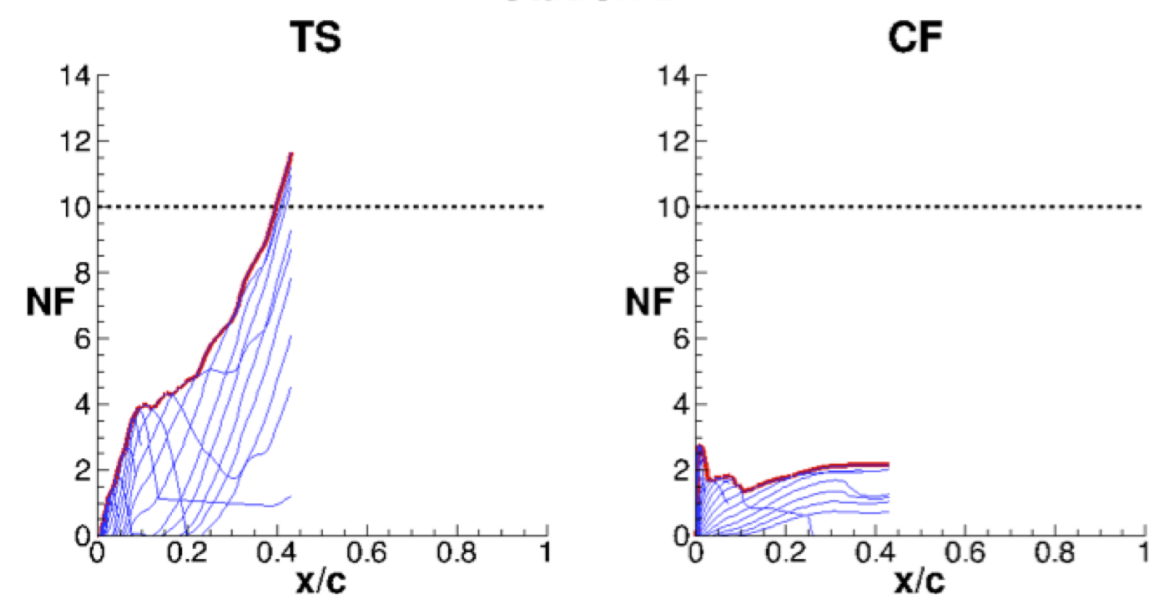

a) Station 2 TS growth (left) and CF growth (right).

\section{Station 4}
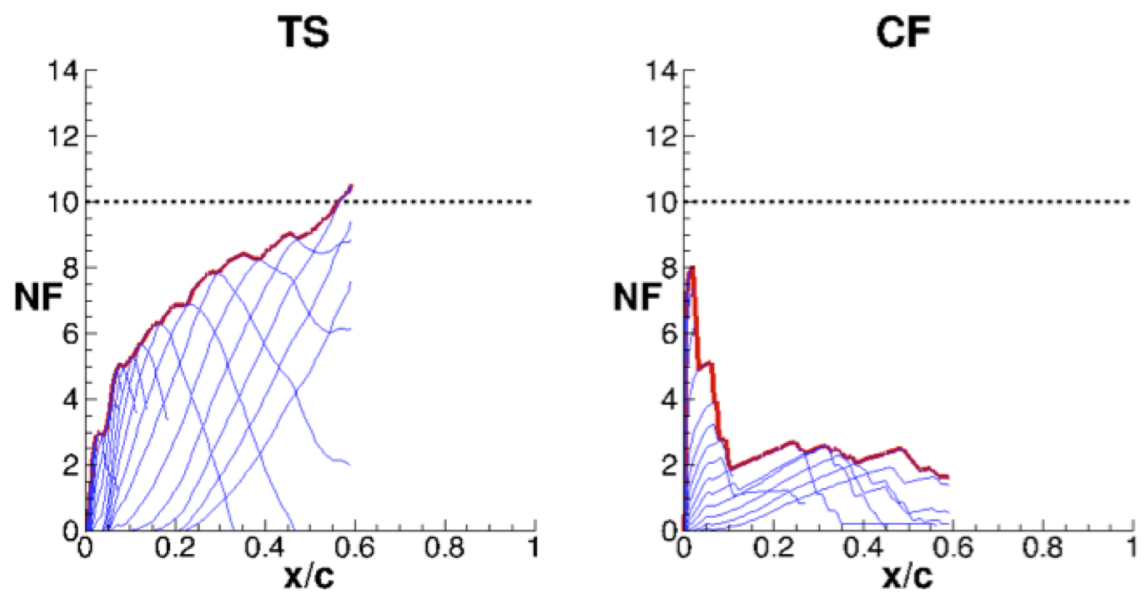

b) Station 4 TS growth (left) and CF growth (right).

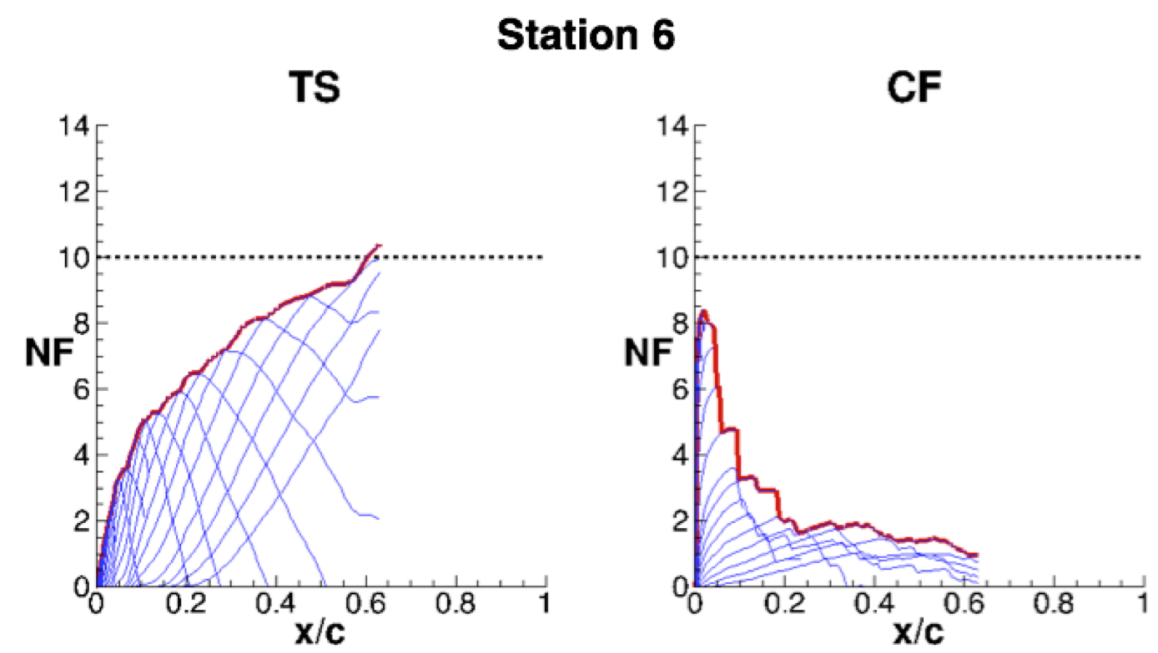

c) Station 6 TS growth (left) and CF growth (right).

Figure 9. Linear stability analysis results showing the NF growth over the chord of spanwise stations, including TS (left) and CF (right) instabilities, analyzed at $M=0.85, C_{L}=0.5, \operatorname{Re}_{\operatorname{mac}}=30 \times 10^{6}$. 

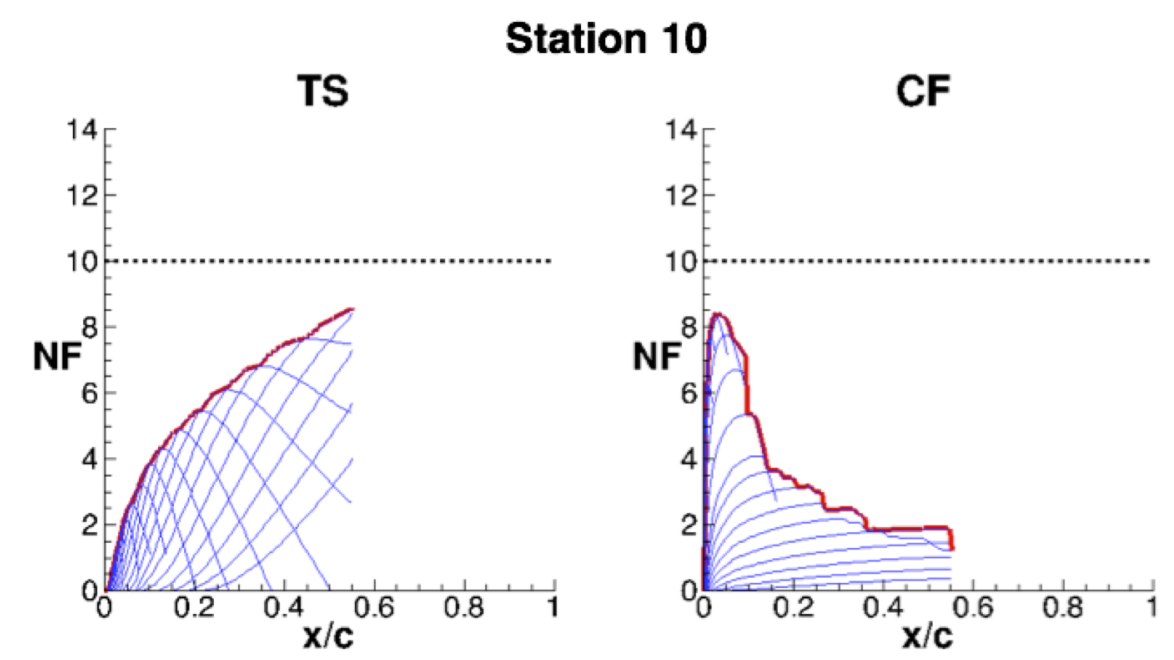

a) Station 10 TS growth (left) and CF growth (right).

Station 12
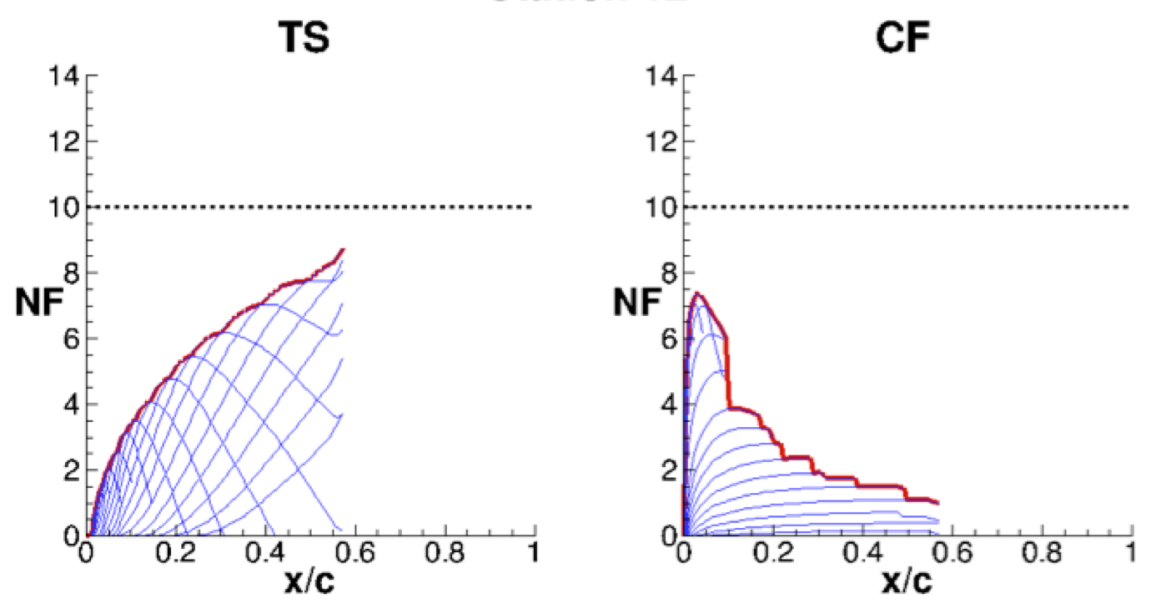

b) Station 12 TS growth (left) and CF growth (right).

\section{Station 14}
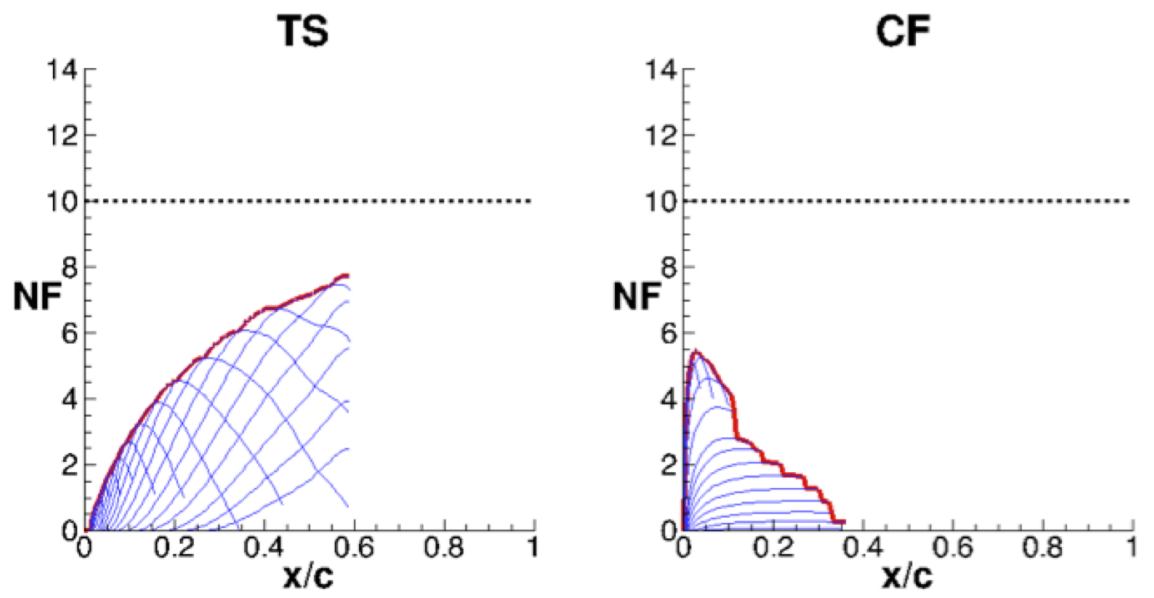

c) Station 14 TS growth (left) and CF growth (right).

Figure 9 (continued). Linear stability analysis results showing the NF growth over the chord of spanwise stations, including TS (left) and CF (right) instabilities, analyzed at $M=0.85, C_{L}=0.5, \operatorname{Re}_{\text {mac }}=30 \times 10^{6}$. 
Additional comparisons of spanwise characteristics between the Baseline and the NLF Design configurations are made in Figure 11. The two configurations were analyzed at the design total lift coefficient of 0.5 ; the angle of attack values needed to match lift coefficient were 2.0 and 2.2 degrees for the Baseline and NLF Design, respectively. As previously mentioned, the NLF Design sectional lift and pitching moment coefficients were designed to maintain similar characteristics to the baseline configuration. The small additional aft loading over the inner half of the wing can be seen. The leading-edge radius of the NLF Design is smaller than the Baseline, in order to meet the $\mathrm{Re}_{\theta}$ attachment line requirement as well as achieve the rapid acceleration near the leading edge to control CF growth. The twist was free to adjust as necessary to obtain the target pressures, and twist smoothing was applied toward the end of the design process. The maximum thickness was held constant throughout the design, giving a slight increase in maximum $\mathrm{t} / \mathrm{c}$ over the inboard reduced leading-edge sweep region, but the chordwise location of maximum thickness was free to adjust as necessary.

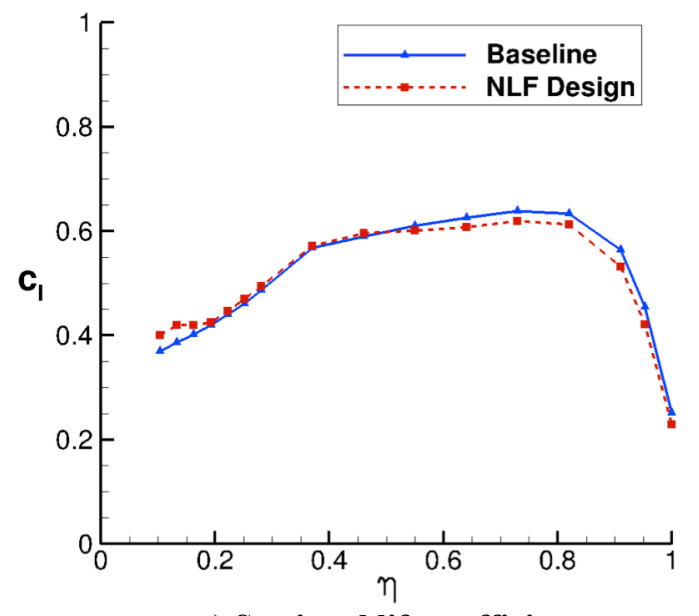

a) Sectional lift coefficient.

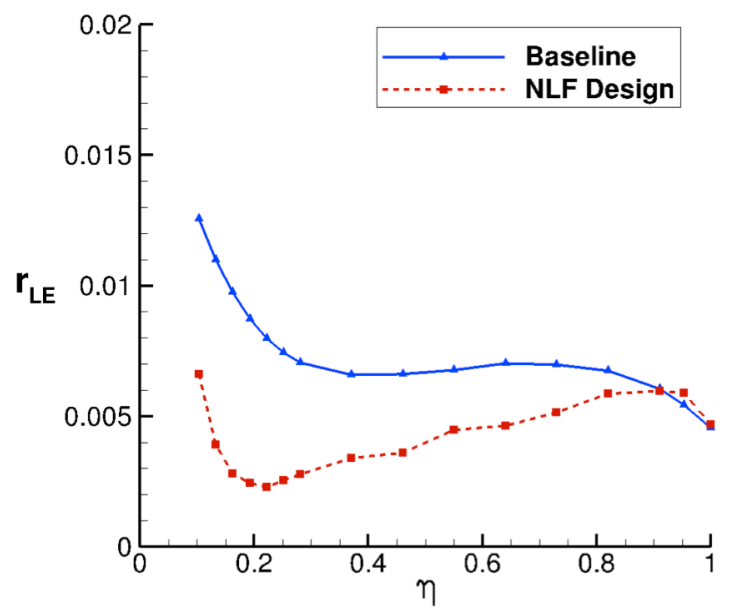

c) Leading-edge radius.

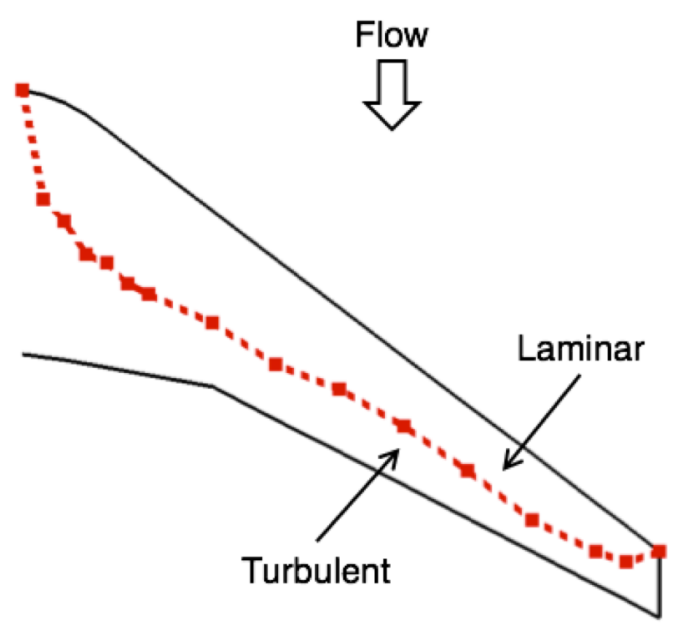

Figure 10. Planform view of the NLF Design wing showing the transition front at the design flight conditions $\left(M=0.85, C_{L}=0.5, \operatorname{Re}_{m a c}=30 \times 10^{6}\right)$.

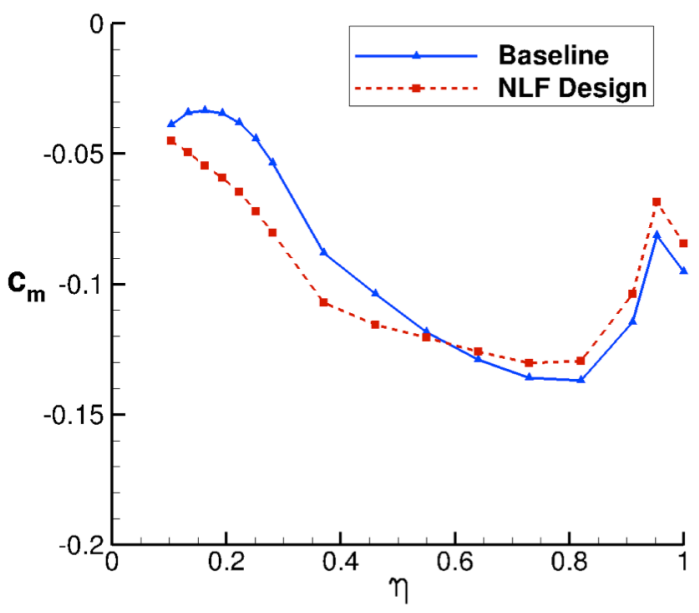

b) Sectional pitching moment coefficient.

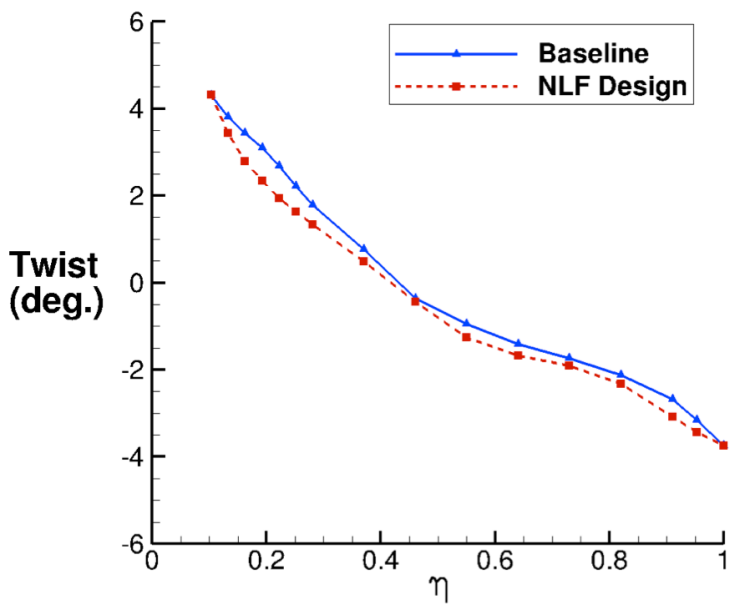

d) Twist in degrees.

Figure 11. Comparison of Baseline (blue, solid) and NLF Design (red, dashed) configurations, showing spanwise characteristics analyzed at the design flight conditions $\left(M=0.85, C_{L}=0.5, R_{m a c}=30 \times 10^{6}\right)$. 


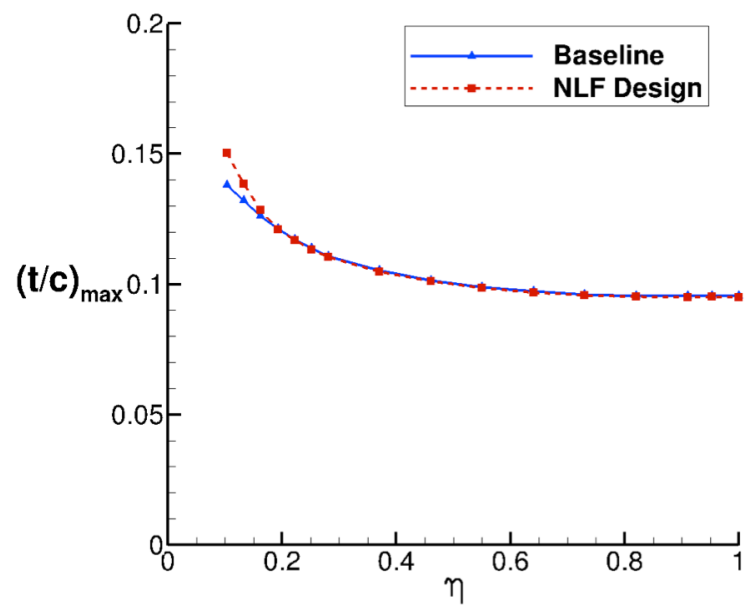

e) Maximum thickness-to-chord ratio.

Figure 11 (continued). Comparison of Baseline (blue, solid) and NLF Design (red, dashed) configurations, showing spanwise characteristics analyzed at the design flight conditions $\left(M=0.85, C_{L}=0.5, \operatorname{Re}_{\text {mac }}=30 \times 10^{6}\right)$.

While performance was not the primary design goal for this wind tunnel model, aerodynamic data was monitored throughout the design process. The Baseline configuration at the design lift coefficient of 0.5 had a drag coefficient of 0.0236 for the wingbody-horizontal tail grid. The final NLF Design configuration supported $56 \%$ laminar flow, which reduced the drag to a drag coefficient of 0.0220 , at the design lift coefficient of 0.5 . The reduction in drag totals to 16 counts, or approximately $6.8 \%$ of the Baseline configuration total drag. In general, it is believed that the drag reduction from sustaining laminar flow would be increased if designing to a higher critical NF because less aggressive TS damping would allow more adverse pressure gradients that could reduce the wave drag penalty. Figure 12 puts these design results in perspective with current NLF/LFC technology. The NLF Design configuration is shown on the transition Reynolds number versus leading-edge sweep plot, with each green triangle representing a different spanwise location from root to tip. With its predicted extents of laminar flow, this NLF wind tunnel model could significantly expand the current boundary of NLF.

\section{Off-Design Results}

When designing a wing that relies on laminar flow for performance, it is important to evaluate the penalty of losing the laminar flow. To evaluate this penalty, the geometry was analyzed as a fully turbulent configuration in USM3D. This analysis technique represents a worst-case scenario where all laminar flow is lost. The effects on pressure distributions can be seen in Figure 13. The NLF Design with laminar flow (red, dashed) and the NLF Design analyzed fully turbulent (green, dash-dot) are taken from flow solutions where the total lift coefficient is at the design condition of 0.5. The NLF Design analyzed fully turbulent required an increase in angle of attack of 0.4 degrees in order to recover the lift lost from losing laminar flow. The pressure distributions show a shift in shock location and strength, as well as a difference in aft loading between the laminar and turbulent analysis of the NLF Design configuration. The turbulent analysis had an increase in drag from the analysis with laminar flow, which is expected as the configuration no longer has the laminar flow related benefits such as reduced skin friction or reduced boundary layer thickness. 


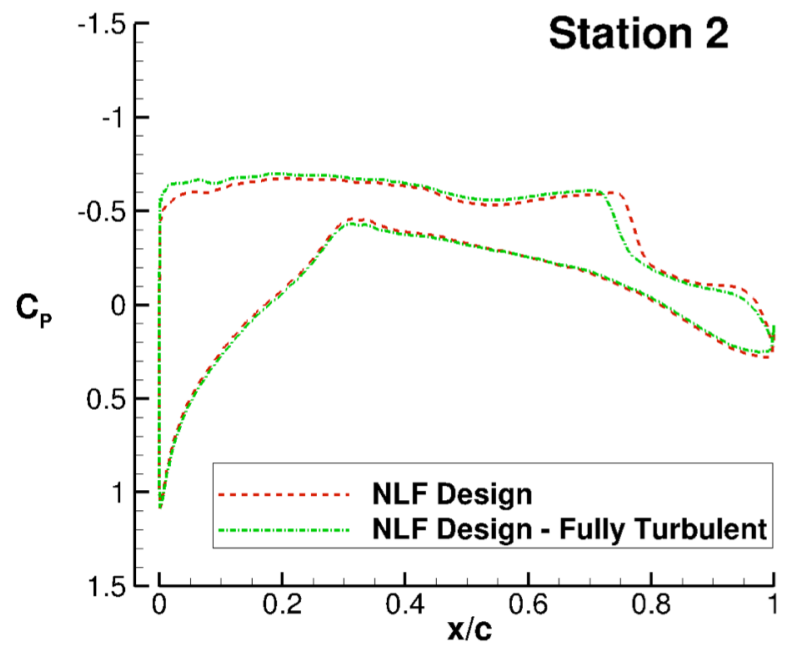

a) Station 2 .

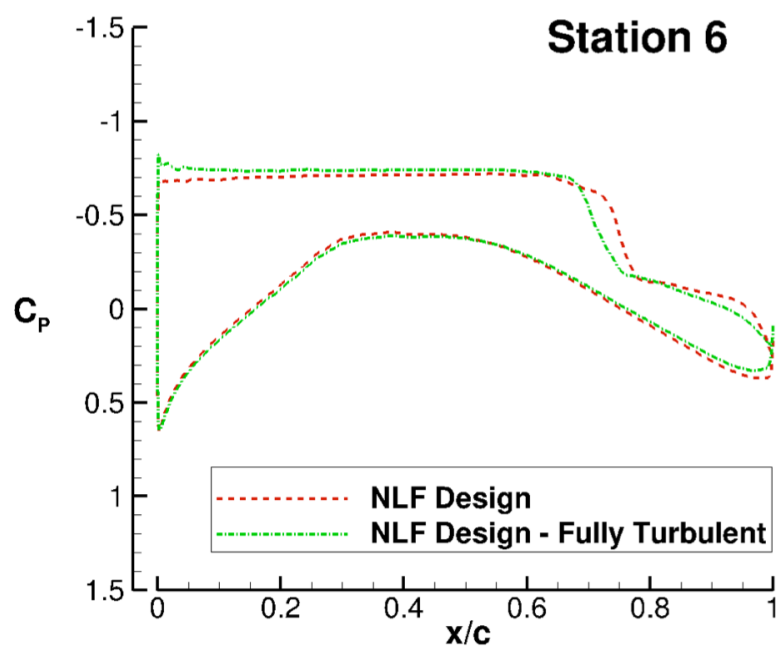

c) Station 6.

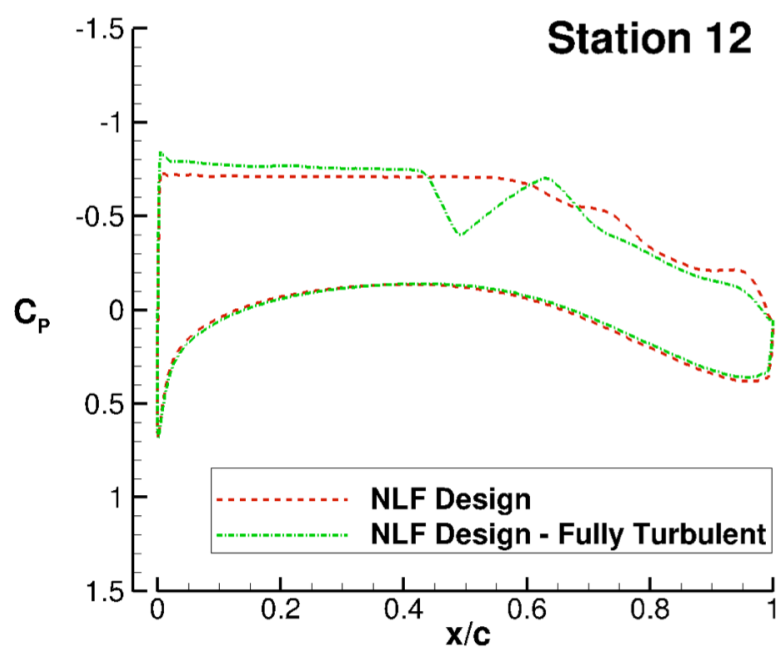

e) Station 12 .

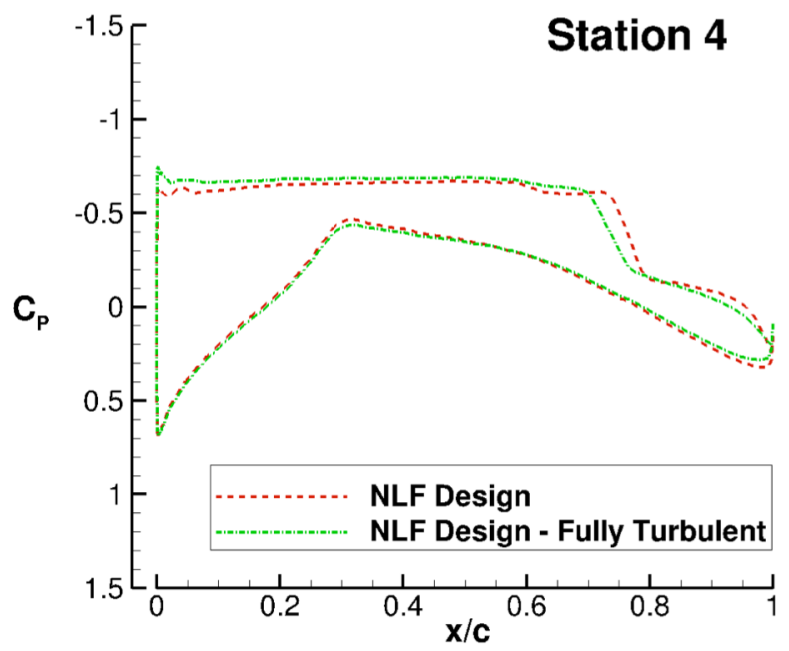

b) Station 4 .

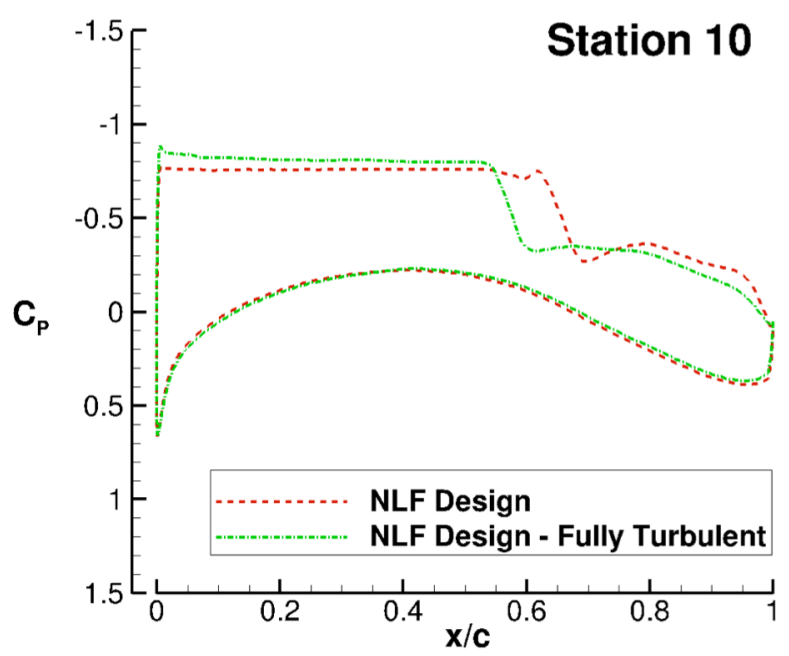

d) Station 10 .

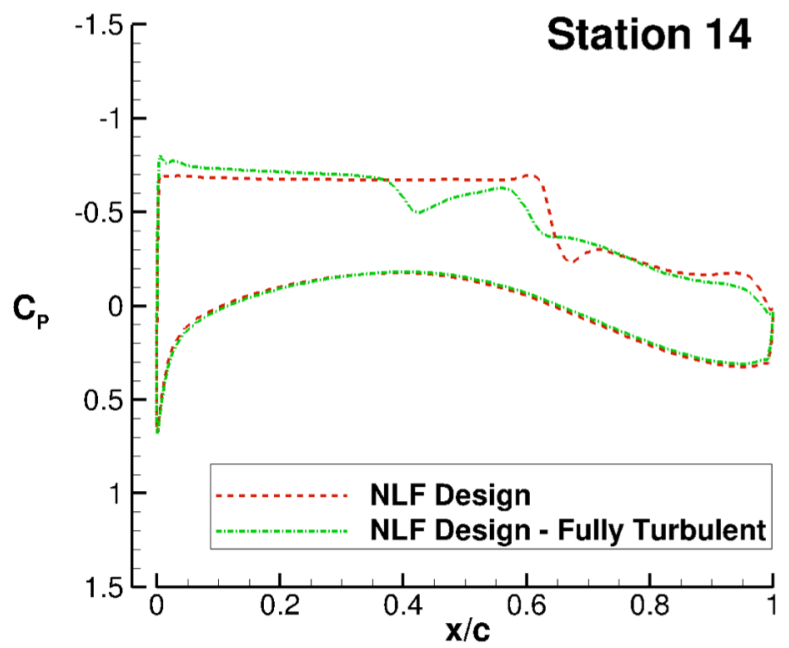

f) Station 14 .

Figure 13. Pressure distributions comparing the NLF Design analyzed with laminar flow (red, dashed) and analyzed fully turbulent (green, dash-dot), both at $M=0.85, C_{L}=0.5, \operatorname{Re}_{\operatorname{mac}}=30 \times 10^{6}$. 
Table 2 presents the drag coefficients from the three analyses for comparison. This table shows that the fully turbulent NLF Design incurred a $4.2 \%$ penalty from the turbulent Baseline to support the NLF capability. Reducing the penalty between the Baseline and fully turbulent NLF Design in future designs would help make NLF a practical application for production vehicles. The penalty of going from laminar to turbulent is inherent in laminar flow technology, but can perhaps be viable if the turbulent configuration performed as well as the Baseline.

Table 2. Drag differences seen at the design flight conditions $\left(M=0.85, C_{L}=0.5, \operatorname{Re}_{\operatorname{mac}}=30 \times 10^{6}\right)$.

\begin{tabular}{|c|c|c|c|}
\hline Configuration & $\mathbf{C}_{\mathbf{L}}$ & $\mathbf{C}_{\mathbf{D}}$ & Difference from Baseline \\
\hline Baseline & 0.500 & 0.0236 & \\
\hline NLF Design & 0.500 & 0.0220 & -16 counts $(6.8 \%$ savings $)$ \\
\hline NLF Design - Fully Turbulent & 0.500 & 0.0246 & +10 counts $(4.2 \%$ penalty) \\
\hline
\end{tabular}

In addition to evaluating the penalty of losing all laminar flow, a near-cruise analysis was conducted to determine how small changes in angle of attack affect the predicted extents of laminar flow. A drag polar showing all three analyses is shown in Figure 14. The polar on the left shows the complete angle of attack range analyzed, which was $1-3$ degrees in increments of 0.25 degrees. The polar on the right is zoomed in to better illustrate $+/-10 \%$ of the design lift coefficient, which represents the cruise range of the vehicle. The laminar NLF Design curve was obtained by iterating between flow solver and stability analyses three times for each point in order to ensure the correct extent of laminar flow was accounted for in the force data. The polar shows the NLF Design has a sustained performance benefit throughout the angle of attack range analyzed. The fully turbulent NLF Design confirmed that there is a penalty when losing laminar flow, and also that there is a turbulent penalty when compared to the turbulent Baseline, although that penalty appears to decrease at lower angles of attack.
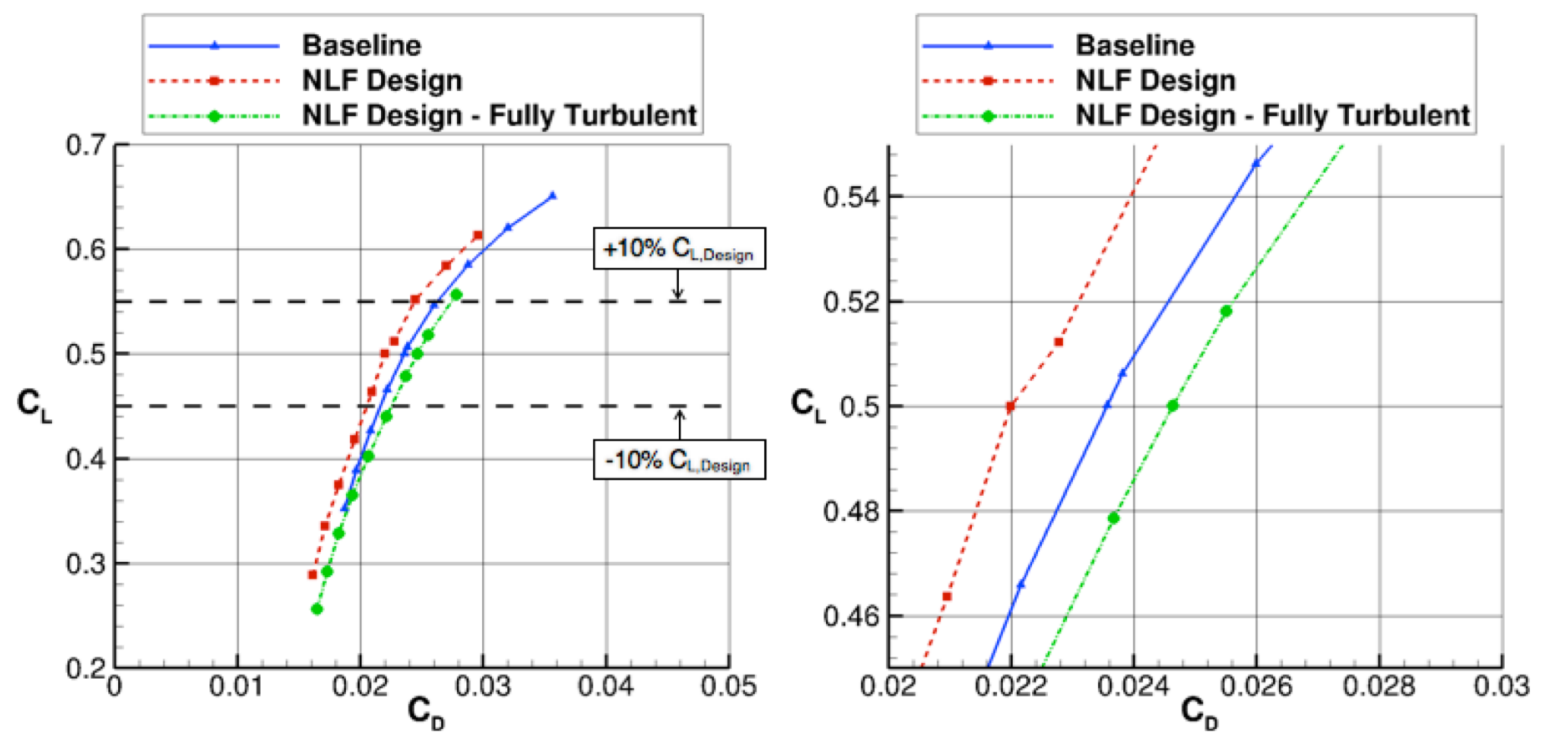

Figure 14. Drag polar comparing the Baseline turbulent configuration (blue, solid) against the NLF Design analyzed with laminar flow (red, dashed) and fully turbulent (green, dash-dot). Left shows the full angle of attack range of 1-3 degrees. Right shows the $+/-10 \%$ design lift coefficient in more detail to highlight the performance at the near-cruise range. Both polars are at design flight conditions $\left(M=0.85, \operatorname{Re}_{\text {mac }}=30 \times 10^{6}\right)$.

The off-design effects on attachment line state can be seen in Figure 15. The inboard reduced sweep section continued to produce $\mathrm{Re}_{\theta}$ values below 100 at both the $+/-10 \%$ design lift conditions, although the extent of lower $\operatorname{Re}_{\theta}$ was reduced when going up in angle of attack. Figure 16 shows how the transition front changes with these small changes of angle of attack. The majority of laminar flow extent is maintained throughout the cruise range of $+/-10 \%$ of the design lift coefficient.

The CDISC NLF design method was successfully applied to the CRM geometry to support significant extents of NLF on the wing upper surface. The technique addressed Görtler vortices, attachment line contamination and transition, and CF and TS modal instabilities for a flight environment critical NF of 10. The extents of laminar flow are predicted to be present even at off-design conditions. During the proposed wind tunnel test in the NTF, the tunnel environment is expected to have a much lower critical NF than the flight environment the model was designed for. A 
testing strategy has been developed to accommodate the lower critical NF to satisfy the wind tunnel test goals with this NLF model and is described in the next section.

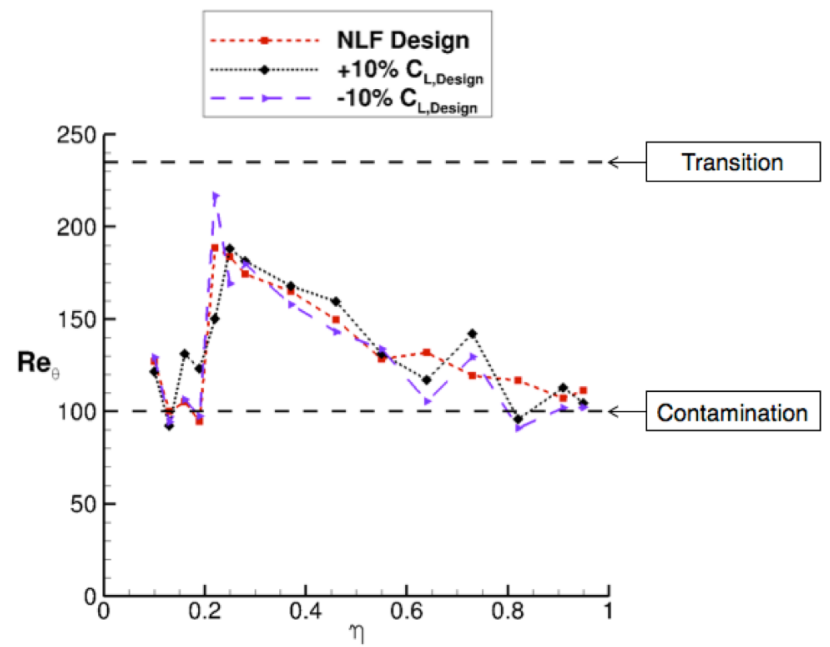

Figure 15. Off-design effects on attachment line state.

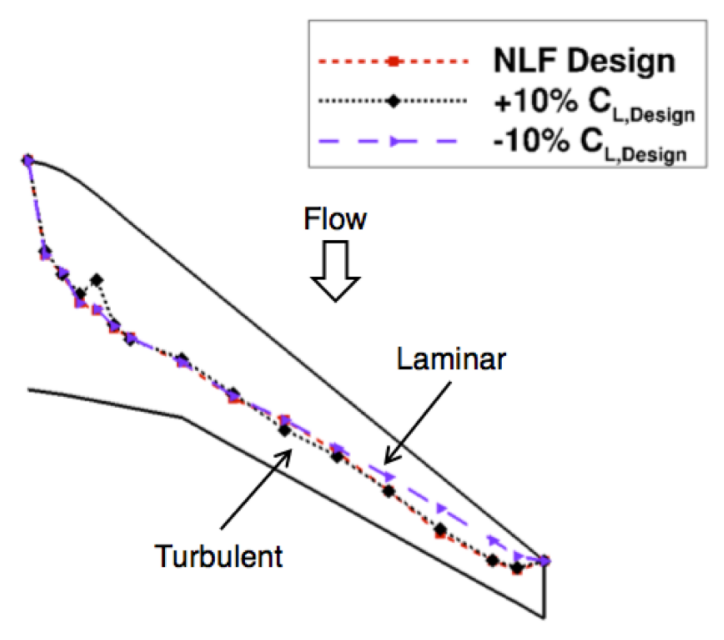

Figure 16. Off-design effects on transition front.

\section{Testing Strategy for the NLF Wind Tunnel Test}

The proposed wind tunnel test will take place in the National Transonic Facility (NTF) at the NASA Langley Research Center. The NTF is a high-pressure, cryogenic, closed-circuit wind tunnel that uses supercold nitrogen gas at high pressure to duplicate true flight aerodynamics. It is one of a very few wind tunnels in the world capable of reaching flight Reynolds numbers. The new design method predicts NLF at Reynolds numbers much higher than previously observed, so it is essential that the test environment is able to reach those high Reynolds numbers in order to validate these predictions.

One of the primary goals for the proposed NLF wind tunnel test is to better identify the facility's laminar flow testing capabilities. The aim is to design and build the model in a way that allows for quantifying different tunnel characteristics, such as the TS and CF critical NF values. As previously mentioned, the strategy for this NLF model was to design to a conservative flight critical NF in order to avoid incurring performance penalties and unrealistic geometries that are associated with designing to a low tunnel critical NF. In this case, the model was designed to a critical NF of 10, and the wind tunnel environment has an estimated critical NF of 6.

The effect that these different environments have on the transition front is shown in Figure 17. While approximately $56 \%$ of the surface area of the original flight design sustained laminar flow, the stability analysis predicts that only two small patches of laminar flow would be present in the wind tunnel at the design Reynolds number of 30 million. The majority of the wing in the wind tunnel is predicted to transition due to $\mathrm{CF}$ instabilities very near the leading edge. Both patches of laminar flow seen at this condition have characteristics that suppress the $\mathrm{CF}$ instabilities: the inboard patch of laminar flow has reduced leading-edge sweep and the outboard patch of laminar flow has reduced chord Reynolds number. These patches of laminar flow offer the unique opportunity to characterize the wind tunnel facility because they isolate transition due to TS and CF. Within the laminar flow patches, transition is midchord and due to TS growth. Therefore, an analysis of chordwise transition location at stations inside the laminar flow patch can be used to identify the TS critical NF. The proximity of transition due to $\mathrm{CF}$ near the leading edge

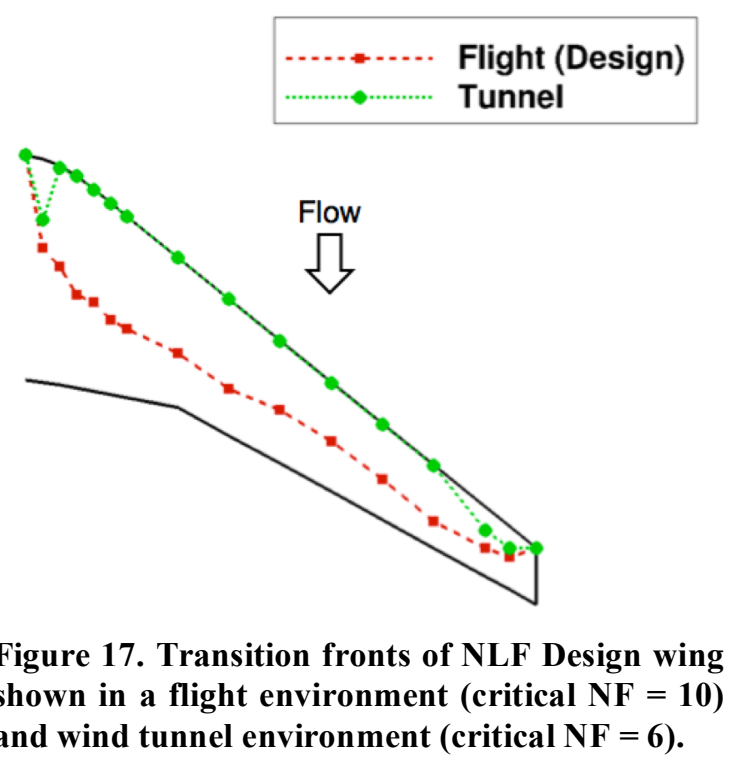

16

American Institute of Aeronautics and Astronautics 


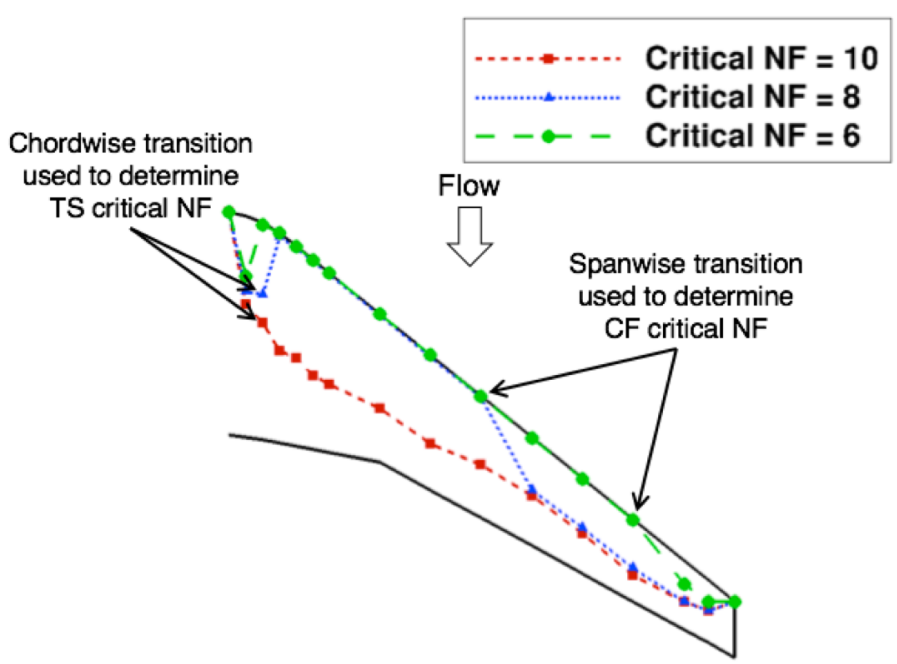

Figure 18. Transition fronts of the NLF Design wing analyzed at a variety of critical $\mathrm{NF}$ at the design Mach of 0.85 and Reynolds number of 30 million. The spanwise and chordwise locations of laminar flow observed during the test will be used to determine NTF critical NF for TS and CF.

flow testing ability will be valuable for future laminar flow tests in the facility.

In addition to quantifying the NTF critical NF for both CF and TS, the second primary goal of the test is to confirm predicted extents of laminar flow, which will help validate the CDISC NLF design method. Larger regions of laminar flow are useful for confirming predictions. Reducing the Reynolds number during the test will provide the additional instability damping needed to sustain laminar flow on the NLF Design in the tunnel environment. Figure 19 shows the effects of reducing Reynolds number on the transition front. The analysis was performed at the predicted tunnel critical NF of 6 . The corresponding reduction in attachment line $\mathrm{Re}_{\theta}$ due to reducing the test Reynolds number is shown in Figure 20. During the test, the tunnel will need to be operating at constant dynamic pressure in order to ensure the model is experiencing similar loads and wing deformations at the different Reynolds numbers. The reduction of test Reynolds number will also help identify if the tunnel critical NF is sensitive to tunnel run conditions. Additionally, the number of turbulent wedges that appear in transition images due to particulates in the tunnel increases with test Reynolds number. Altering the test Reynolds number will be helpful in identifying any laminar flow testing limits due to flow quality in the NTF.

The wind tunnel model will be a semispan 5.2\% scale model of the NLF Design geometry. The model is semispan in order to maximize the chord length, which will reduce the required unit Reynolds number during the test. This will increase the boundary layer thickness, which reduces the model's sensitivity to particles in the wind tunnel and to surface imperfections. During the test, the wing will deform due to aeroelastic effects, so the model will be built to an unloaded jig-shape such that the wing deforms into the design shape when the tunnel is operating. The jig-shape is unloaded based on material properties, tunnel conditions, and the expected loads at a specific test condition, most often the design condition. However, for a NLF wing, the extent of laminar flow greatly influences the loads, as seen in the offdesign analysis of the fully turbulent wing. For the significant extents of NLF seen on this model, the loads change makes determining the CF critical NF by a impractical. The transition images from the wind tunnel are not expected to have the resolution necessary to determine the chordwise CF transition location due to the leading-edge curvature of the model. However, because the two laminar flow hes only exist when the $\mathrm{CF}$ instabilities critical NF, the spanwise location of laminar flow patches can help identify this tunnel characteristic. The tunnel CF critical NF can be determined by analyzing stations slightly ind slightly outboard of the spanwise Figure 18 shows the transition fronts of the NLF Design analyzed at several critical NF values at the design Reynolds number of 30 million. This image highlights that both chordwise and spanwise analyses can be used to determine the tunnel TS and CF critical NF, respectively. Quantifying the NTF laminar

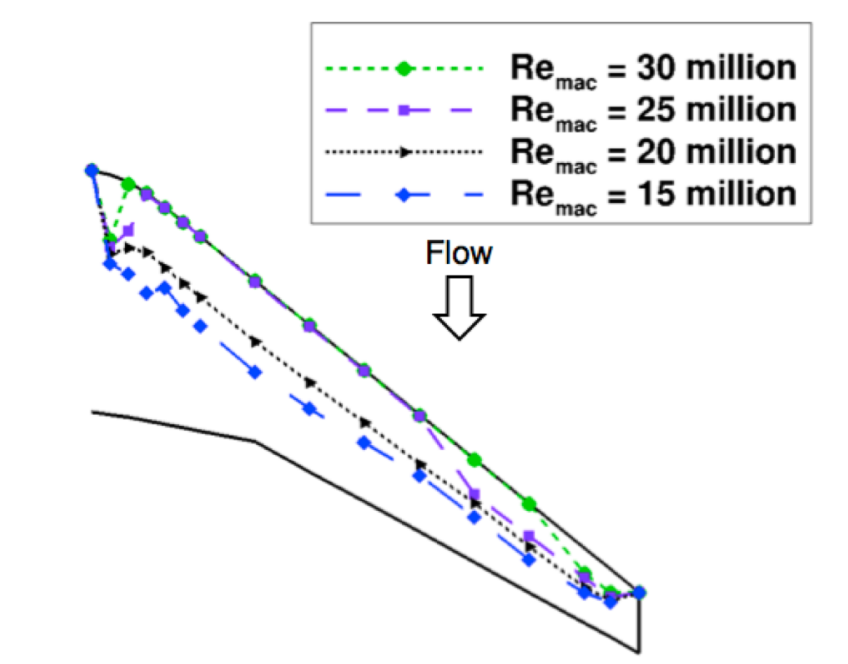

Figure 19. Transition fronts of the NLF Design wing analyzed at $M=0.85$ in the wind tunnel environment (critical $\mathrm{NF}=6$ ) at a variety of Reynolds numbers. 


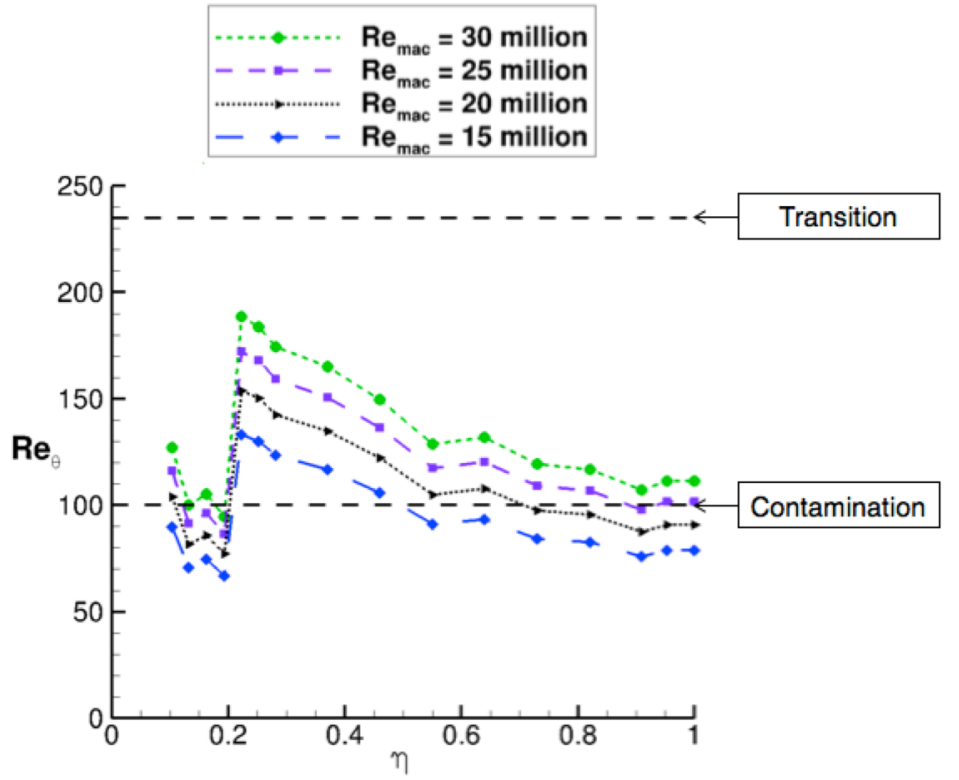

Figure 20. Reynolds number effects on attachment line state. approximately $10 \%$ by losing laminar flow. This requires the jig-shape to be built based on loads that assume a specific laminar flow extent. Predicting the extent of laminar flow presents a unique challenge for building NLF models, since the laminar flow extent is highly dependent on the tunnel environment, which is often unknown until the model is in the tunnel. The wing for the upcoming NTF test was designed for flight conditions of 30 million Reynolds number and a critical NF of 10, but the wind tunnel model jig-shape will be built for the loads predicted at a Reynolds number of 20 million and a critical NF of 6 . Based on previous laminar flow tests in the NTF, 20 million Reynolds number with a critical NF of 6 seemed a more reasonable condition to expect significant extents of laminar flow in the wind tunnel environment. The nearcruise analysis suggested that small changes in angle of attack have little effect on the extents of laminar flow. While the model

will be built for the loads at one condition, the changes in deformation due to varying extents of laminar flow is assumed to be small enough to not greatly impact the laminar flow. Historically, pressure taps are avoided on a laminar flow model in order to eliminate premature transition due to the taps. However, since this model is semispan, the only way to get the necessary pressure information is through pressure taps on the laminar flow surface. To decrease the chances of tripping the flow, the pressure orifices near the leading edge will be smaller $(0.01$ " diameter in the forward 0.20 chord, 0.02 " diameter aft) and the pressure tubing will not pierce the surface. The semispan model will be fitted with model deformation targets and 230 static pressure taps on the upper and lower surfaces to ensure adequate data on the deformation and resulting pressure distributions is acquired during the test.

Another important aspect of laminar flow testing is establishing best practices relating to manufacturing tolerance, surface finish, and visualization techniques. The proposed NLF test in the NTF will be used to better identify these best practices. Manufacturing tolerances are important to reduce the difference between the computationally-based design and the physical model. For this model, the manufacturing tolerances are increasingly important near the leading edge, since obtaining the design pressure distributions in that region is critical to avoiding attachment line transition or contamination and to damping CF instabilities. It has been requested the wing match the design geometry within \pm 0.003 inch or better for surface contour tolerance, with increased tolerance requirements near the leading edge. Another critical item of a NLF model is surface finish. Poor surface finish quality can cause turbulent wedges and significantly limit the extents of laminar flow seen during the test. However, getting a model to the surface finish typically required in a laminar flow test involves extensive polishing, which significantly increases the model cost and extends the manufacturing timeline. For the upcoming test in the NTF, it has been requested that the wing be built to a surface finish of a standard NTF model of 16 micro-inch Root Mean Square (RMS). The additional polishing needed to sustain laminar flow in the NTF will be obtained through polishing the paint layer used for laminar flow visualization. It has been requested that this model have a final surface finish between 2-4 micro-inch RMS. The proposed visualization technique for this test is a temperature sensitive paint (TSP) layer using carbon nanotubes (CNT) as the temperature gradient mechanism. TSP has been used in several laminar flow tests previously ${ }^{17}$, and it requires a temperature difference between the model and the tunnel flow in order to show transition. This temperature gradient is traditionally achieved with a burst of liquid nitrogen to cool the tunnel flow quickly. However, this burst of liquid nitrogen alters the tunnel flow conditions, creating a dynamic transition front due to the changing conditions. Additionally, injecting liquid nitrogen for the temperature gradient is costly and less efficient. For the proposed NTF test, a CNT layer will be used to heat the model quickly, providing the necessary temperature gradient ${ }^{18}$. The CNT layer is conductive and produces a uniform electrical heating when power is applied to the layer. The CNT layer is expected to improve the transition data by maintaining tunnel conditions while acquiring the transition image, as well as eliminate the costs and efficiency penalties associated with a rapid liquid nitrogen injection. As mentioned, this TSP/CNT layer will be polished to meet the surface finish requirements of laminar flow testing. 
The proposed test in the NTF has three primary test goals to advance the current laminar flow testing capabilities. The test will be used to calculate the critical NF of both CF and TS in the NTF at various conditions. Additionally, the extents of laminar flow observed during the test will validate the CDISC NLF design method. The test will also help establish best practices for future laminar flow testing, relating to manufacturing, surface finish, and visualization techniques.

\section{Conclusion}

Natural laminar flow (NLF) is a technology that has the potential to significantly improve the performance of a vehicle. Current NLF technology is limited to aircraft components with lower Reynolds number and smaller leadingedge sweep, such as nacelles, winglets, or wings with low sweep. A new computationally-based NLF design method has been developed that predicts significant extents of NLF on a wing with high sweep and high Reynolds numbers. A crucial step toward incorporating this technology into flight vehicles is validating designs in wind tunnels. The differences in turbulence levels experienced in flight and wind tunnel environments creates a unique challenge for laminar flow testing.

A transonic wind tunnel model has been designed using the new NLF design capability. The baseline configuration is the Common Research Model (CRM), with sweep and Reynolds numbers seen on typical transports and has been studied both computationally and experimentally in the past. The NLF design is predicted to sustain significant extents of laminar flow; at the design condition, the upper surface of the wing supports 56\% laminar flow. Off-design analysis shows the vehicle in a fully-turbulent state experiences performance penalties compared to both the configuration with laminar flow and the turbulent baseline CRM. The model is predicted to maintain significant extents of laminar flow through an angle of attack range equivalent to near-cruise conditions. The predicted extents of NLF would greatly expand the current limits of NLF relating to leading-edge sweep and transition Reynolds number.

A semispan model of the NLF design is being built to be tested in the National Transonic Facility (NTF) at the NASA Langley Research Center. The test goals include quantifying the facility's laminar flow testing capabilities, including estimating critical $\mathrm{N}$-factors and identifying any upper Reynolds number limits on flow quality. The test will also validate the computational tools and methods used in designing the model. Best practices will be more clearly identified for future laminar flow testing, including requirements on manufacturing tolerances, surface finish, and visualization techniques. It is hoped that the NTF laminar flow test will offer insight to improve the laminar flow testing techniques and confirm that NLF can be sustained on wings with high sweep and high Reynolds numbers.

\section{Acknowledgments}

This research is funded by the NASA Advanced Air Transport Technology Project within the Advanced Air Vehicles Program. The authors would like to thank Dr. Ponnampalam Balakumar, Dr. Chau-Lyan Chang, Dr. Meelan Choudhari, and Dr. Lewis Owens of the NASA Langley Research Center for offering their support and expertise in boundary layer stability computations. Additionally, the authors would like to thank the test planning team for their contribution to the design of the wind tunnel model.

\section{References}

${ }^{1}$ Anderson, B. T., and Meyer, R. R., "Effects of Wing Sweep on In-Flight Boundary Layer Transition for a Laminar Flow Wing at Mach Numbers from 0.60 to 0.79 ," NASA TM-101701, 1990.

${ }^{2}$ Fujino, M., "Design and Development of the HondaJet", AIAA International Air and Space Symposium and Exposition: The Next 100 Years, AIAA 2003-2530, July 2003.

${ }^{3}$ Crouch, J., "Boundary-Layer Transition Prediction for Laminar Flow Control (Invited)", 45th AIAA Fluid Dynamics Conference, AIAA AVIATION Forum, AIAA 2015-2472, 2015.

${ }^{4}$ Frink, N.T., Pirzadeh, S.Z., Parikh, P.C., Pandya, M.J., and Bhat, M.K., "The NASA Tetrahedral Unstructured Software System," The Aeronautical Journal, Vol. 104, No. 1040, October 2000, pp.491-499.

${ }^{5}$ Campbell, R.L., "Efficient Viscous Design of Realistic Aircraft Configurations," AIAA-98-2539, June 1998.

${ }^{6}$ Campbell, R.L., and Lynde, M.N., "Natural Laminar Flow Design for Wings with Moderate Sweep", 34th AIAA Applied Aerodynamic Conference, AIAA AVIATION Forum, AIAA 2016-4326, June 2016.

${ }^{7}$ Lynde, M.N. and Campbell, R.L., "Expanding the Natural Laminar Flow Boundary for Supersonic Transports", 34th AIAA Applied Aerodynamic Conference, AIAA AVIATION Forum, AIAA 2016-4327, June 2016.

${ }^{8}$ Wie, Y.-S., "BLSTA: A Boundary Layer Code for Stability Analysis," NASA CR 4481, 1992.

${ }^{9}$ Chang, C.-L., "The Langley Stability and Transition Analysis Code (LASTRAC): LST, Linear and Nonlinear PSE for 2-D, Axisymmetric, and Infinite Swept Wing Boundary Layers," AIAA 2003-0974, 2003.

${ }^{10}$ Poll, D. I. A., "Some Observations of the Transition Process on the Windward Face of a Long Yawed Cylinder," J. Fluid Mech., Vol. 150, 1985, pp. 329-356.

${ }^{11}$ Joslin, R. D., "Overview of Laminar Flow Control," NASA TP-1998-208705, 1998. 
${ }^{12}$ Gatlin, G.M., Parker, P.A., Owens, L.R., "Development of a Semi-Span Test Capability at the National Transonic Facility (Invited)", AIAA 2001-0759, 39th AIAA Aerospace Sciences Meeting and Exhibit, January 2001.

${ }^{13}$ Vassberg, J.C. and Rivers, S.M., "Development of a Common Research Model for Applied CFD Validation", AIAA 2008-6919, August 2008.

${ }^{14}$ Rivers, M. and Dittberner, A., "Experimental Investigations of the NASA Common Research Model (Invited)", 28th AIAA Applied Aerodynamics Conference, Fluid Dynamics and Co-located Conferences, AIAA 2010-4218, June 2010.

${ }^{15}$ Crouch, J., Sutanto, M., Witkowski, D., Watkins, A., Rivers, M., and Campbell, R., "Assessment of the National Transonic Facility for Natural Laminar Flow Testing", 48th AIAA Aerospace Sciences Meeting Including the New Horizons Forum and Aerospace Exposition, Aerospace Sciences Meetings, AIAA 2010-1302, January 2010.

${ }^{16}$ Belisle, M.J., Roberts, M.W., Williams, T.C., Tufts, M.W., Tucker, A.A., Saric, W.S., and Reed, H.L., "A Transonic LaminarFlow Wing Glove Flight Experiment: Overview and Design Optimization”, AIAA 2012-2667, June 2012.

${ }^{17}$ Hammer, M., Popernac, T., Owens, L., and Wahls, R., "Using temperature sensitive paint technology", 40th AIAA Aerospace Sciences Meeting \& Exhibit, Aerospace Sciences Meetings, AIAA 2002-0742, 2002.

${ }^{18}$ Klein, C. and Henne, U., "Combination of Temperature Sensitive Paint and Carbon Nanotubes for Transition Detection", 53rd AIAA Aerospace Sciences Meeting, AIAA SciTech, AIAA 2015-1558, January 2015. 